\title{
Switching Boundary Conditions in the Many-Body Diffusion Algorithm
}

\author{
F. Luczak, F. Brosens * and J.T. Devreese ${ }^{\dagger}$ \\ Departement Natuurkunde, Universiteit Antwerpen (UIA), \\ Universiteitsplein 1, B-2610 Antwerpen \\ L. F. Lemmens \\ Departement Natuurkunde, Universiteit Antwerpen (RUCA), \\ Groenenborgerlaan 171, B-2020 Antwerpen
}

(January 27, 1998)

\begin{abstract}
In this paper we show how the transposition, the basic operation of the permutation group, can be taken into account in a diffusion process of identical particles. Whereas in an earlier approach the method was applied to systems in which the potential is invariant under interchanging the Cartesian components of the particle coordinates, this condition on the potential is avoided here. In general, the potential introduces a switching of the boundary conditions of the walkers. [These transitions modelled by a continuous-time Markov chain generate sample paths for the propagator as a Feynman-Kac functional. A few examples, including harmonic fermions with an anharmonic interaction, and the ground-state energy of ortho-helium are studied to elucidate the theoretical discussion and to illustrate the feasibility of a sign-problem-free implementation scheme for the recently developed many-body diffusion approach.]
\end{abstract}

\section{INTRODUCTION}

In a previous paper [1], the present authors have shown that the many-body diffusion algorithm (MBDA) allows for a sign-problem-free simulation of excited antisymmetric states of interacting harmonic oscillators. The basic idea behind this approach has been to split the Euclidean-time propagator into a sum of independent propagators which remain positive on the appropriate state space [2]5]. Each of the elementary propagators could be individually sampled as a diffusion process for distinguishable particles on a state space with absorbing or reflecting boundaries.

In the present paper, the many-body diffusion formalism and its implementation are generalized to allow for the construction of interdependent diffusion processes. In this approach the propagator that governs the Euclidean time evolution is split into two parts: the kinetic part is used to describe the evolution of the system with well-defined boundary conditions, the potential is used for branching and killing and rules transitions from one set of boundary conditions to another set. The evolution is given by a Brownian motion or a random walk, but the boundary conditions can change during the simulation according to a continuous-time Markov process. The transition rates of this process partly or completely derive from the potential which determines also the killing or branching process.

This principle is illustrated with some test models. A limited number of degrees of freedom is considered to avoid large computation times and to allow for a visual control on the probability densities that are generated. The presented algorithm does not integrate smoothly with the previous one for harmonic fermions, in the sense that for this algorithm the interchange of two identical particles is fully under control but in combination with the even permutations it is far from trivial if more than two particles of parallel spin are present.

In the next section, the theoretical background of the method is discussed. In Sec. III, details on the diffusion in a reduced state space are provided. In Sec. IV, a sign-problem-free implementation is developed and applied to two toy models to illustrate the concepts. Subsequently, anisotropic harmonic fermions are studied avoiding normal coordinates in order to elucidate the role of the potential for the transitions between different sets of boundary conditions. Finally, the MBDA is employed to calculate the ground-state energy of ortho-helium. Some concluding remarks are presented in Sec. V.

* Senior Research Associate of the FWO-Vlaanderen.

${ }^{\dagger}$ Also at the Universiteit Antwerpen (RUCA) and Technische Universiteit Eindhoven, The Netherlands. 


\section{A SIGN-PROBLEM-FREE APPROACH}

In this section, we describe the construction of a Feynman-Kac functional equipped with a process to generate the sample paths over which the exponential containing the interaction has to be averaged. This construction is based on the many body diffusion approach introduced in [4] and illustrated in [1]. The procedure is sign-problem-free in the strict sense, i.e. expectation values in the Feynman-Kac functional are obtained using transition probabilities in a Wiener-Poisson space. A mathematical account on the foundations of these compound processes can be found in [10]. The Wiener space is the support for the diffusion whereas a multivariate Poisson process counts the switches of the boundary condition as will be explained below.

\section{A. Diffusion on a Domain}

Let $\bar{r}$ be a $3 N$-dimensional point in the configuration space $\mathbb{R}^{3 N}$ of $N$ distinguishable particles and assume that the propagator for this system is given by:

$$
K_{D}\left[\bar{r}_{f}, \tau ; \bar{r}_{i}, 0\right]=\sum_{k}\left(\psi_{k}\left(\bar{r}_{i}\right), \exp (-\tau H) \psi_{k}\left(\bar{r}_{f}\right)\right)
$$

where $H$ describes the evolution in Euclidean time of the $\mathrm{N}$ particles. The subscript $D$ denotes that at this stage the particles are still considered to be distinguishable. This propagator can be easily written as a Feynman-Kac functional over all paths starting in $\bar{r}_{i}$ and ending in $\bar{r}_{f}$ a Euclidean time lapse $\tau$ later. The paths are constructed by a $3 N$-dimensional Brownian motion $\{\bar{R}(\tau) ; \tau \geq 0\}$ with variance $\sigma^{2}=\hbar \tau / m$

$$
K_{D}\left[\bar{r}_{f}, \tau ; \bar{r}_{i}, 0\right]=E_{\bar{r}_{i}}\left[I_{\left(\bar{R}(\tau)=\bar{r}_{f}\right)} \exp \left(-\frac{1}{\hbar} \int_{0}^{\tau} V(\bar{R}(\varsigma)) d \varsigma\right)\right]
$$

All integration paths in (2) start in $\bar{r}_{i}$ as indicated by the averaging index $E_{\bar{r}_{i}}$ and satisfy the condition $\bar{R}(\tau)=\bar{r}_{f}$ as denoted by the indicator $I_{\left(\bar{R}(\tau)=\bar{r}_{f}\right)}$. Writing the propagator as an average over a Brownian motion is equivalent to giving the evolution equation and the initial condition:

$$
\begin{gathered}
\frac{\partial}{\partial \tau} K_{D}\left[\bar{r}_{f}, \tau ; \bar{r}_{i}, 0\right]=\left(\frac{\hbar}{2 m} \nabla_{\bar{r}}^{2}-\frac{1}{\hbar} V\left(\bar{r}_{f}\right)\right) K_{D}\left[\bar{r}_{f}, \tau ; \bar{r}_{i}, 0\right] \\
\lim _{\tau \downarrow 0} K_{D}\left[\bar{r}_{f}, \tau ; \bar{r}_{i}, 0\right]=\delta\left(\bar{r}_{f}-\bar{r}_{i}\right)
\end{gathered}
$$

If a propagator containing two indistinguishable particles $j$ and $k$ has to be derived from (11), a projection on the symmetric (anti-symmetric) irreducible representation of the permutation group has to be made for bosons (fermions):

$$
K_{I}\left[\bar{r}_{f}, \tau ; \bar{r}_{i}, 0\right]=\frac{1}{2 !} \sum_{P} \xi^{P} K_{D}\left[P \bar{r}_{f}, \tau ; \bar{r}_{i}, 0\right]
$$

where $P$ represents the permutations of the particles $j, k$ and $\xi= \pm 1$ is chosen according to the particle statistics under consideration. The evolution equation for the projected propagator is given by the same eq. (3), because $\nabla \frac{2}{r}$ as well as the potential $V(\bar{r})$ are invariant under all the permutations $P$. The initial condition obtained from the projection (5) by taking the limit $\tau \downarrow 0$ is not the initial condition that is usually studied if one wants to solve a partial differential equation using probabilistic methods. [11] In order to obtain an initial condition which is given by

$$
\lim _{\tau \downarrow 0} K_{I}\left[\bar{r}_{f}, \tau ; \bar{r}_{i}, 0\right]=\delta\left(\bar{r}_{f}-\bar{r}_{i}\right) \text { with } \quad \bar{r}_{f}, \bar{r}_{i} \in D_{2}^{3} \otimes \mathbb{R}^{3(N-2)}
$$

one has to restrict the configuration space $\mathbb{R}^{3 N}$ to a domain $D_{2}^{3} \otimes \mathbb{R}^{3(N-2)}$. In this domain the identical-particle coordinates $\vec{r}_{j}, \vec{r}_{k}$ are linearly ordered. In $D_{2}$, for example, the $x$-coordinates are ordered as follows: $x_{j} \geq x_{k}$. Introducing a domain implies that one has to specify the boundary conditions; the specification appropriate for bosons or fermions moving freely or interacting harmonically has been proposed in [4] and analyzed in [1]. 


\section{Boundary conditions}

The ordering particles in a three-dimensional space can be performed by ordering the particles with respect to each of the three independent Cartesian directions. This option has been chosen in the previous investigations and led [1] 4 to the construction of an ordered state-space $D$. The symmetric irreducible representation implies four combinations $l=0,1,2,3$. By definition, the $(l=0)$-boundary condition causes reflection at the boundary $\partial D$ in every direction $x, y, z$, whereas for $l=1,2,3$ the boundary $\partial D$ reflects in $x, y, z$-direction and absorbs in the two other two Cartesian directions. For fermions, $l=0$ means absorption at $\partial D$ in all three directions, whereas for $l=1$ absorption occurs in the $x$-direction and reflection in the $y$ - and $z$-direction. Similarly, $l=2$ and $l=3$ correspond to the appropriate absorption and reflection conditions with respect to the $y$ - and $z$-direction. It is clear that in order to study the evolution of the system in Euclidean time one has to give not only the starting point in the domain $D_{2}^{3}$ but also the boundary conditions $l$. The propagator has to contain the information that a state, initially characterized by $\bar{r}_{i}$ and $l$, will end after a Euclidean time lapse $\tau$ in $\bar{r}_{f}$ with boundary conditions $\hat{l}$. The evolution in Euclidean time of this propagator can be studied from its extension to $\mathbb{R}^{3 N}$, which gives

$$
K_{I}\left[\bar{r}_{f}, l, \tau ; \bar{r}_{i}, \hat{l}, 0\right]=\left(\frac{1}{2 !}\right)^{3} \sum_{P_{x}} \xi_{l}^{P_{x}} \sum_{P_{y}} \xi_{l}^{P_{y}} \sum_{P_{z}} \xi_{l}^{P_{z}} K_{D}\left[P_{x} P_{y} P_{z} \bar{r}_{f}, \tau ; \bar{r}_{i}, \hat{l}, 0\right]
$$

where $P_{x}, P_{y}, P_{z}$ symbolize the permutations of the $x, y, z$-coordinates of the two indistinguishable particles $j$ and $k$ and where we imposed the initial condition

$$
\lim _{\tau \downarrow 0} K_{I}\left[\bar{r}_{f}, l, \tau ; \bar{r}_{i}, \hat{l}, 0\right]=\delta_{l, \hat{l}} \delta\left(\bar{r}_{f}-\bar{r}_{i}\right) \quad, \quad \bar{r}_{f}, \bar{r}_{i} \in D_{2}^{3} \otimes \mathbb{R}^{3(N-2)}
$$

The boundary condition $l$ determines the parity $\xi_{l}^{P_{x}}, \xi_{l}^{P_{y}}, \xi_{l}^{P_{z}}$ of the permutations $P_{x}, P_{y}, P_{z}$.

\section{Evolution equations}

A potential $V(\bar{r})$ whose decomposition contains only one-dimensional irreducible representations with respect to permutations $Q_{x}, Q_{y}, Q_{z}$ of the coordinates is straightforward to analyze. Expanding the potential energy in the possible symmetry combinations, described above, one may write:

$$
V(\bar{r})=\sum_{l^{\prime}=0}^{3}\left(\frac{1}{2 !}\right)^{3} \sum_{Q_{x}} \xi_{l^{\prime}}^{Q_{x}} \sum_{Q_{y}} \xi_{l^{\prime}}^{Q_{y}} \sum_{Q_{z}} \xi_{l^{\prime}}^{Q_{z}} V\left(Q_{x} Q_{y} Q_{z} \bar{r}\right)
$$

Consider now the following decomposition

$$
\begin{aligned}
\chi & =\left(\frac{1}{2 !}\right)^{3} \sum_{P_{x}} \sum_{P_{y}} \sum_{P_{z}} \xi_{l}^{P_{x}} \xi_{l}^{P_{y}} \xi_{l}^{P_{z}} V\left(P_{x} P_{y} P_{z} \bar{r}_{f}\right) K_{D}\left[P_{x} P_{y} P_{z} \bar{r}_{f}, \tau ; \bar{r}_{i}, \hat{l}, 0\right] \\
& =\left(\frac{1}{2 !}\right)^{6} \sum_{l^{\prime}} \sum_{P_{x} Q_{x}} \sum_{P_{y} Q_{y}} \sum_{P_{z} Q_{z}} \xi_{l}^{P_{x}} \xi_{l^{\prime}}^{Q_{x}} \xi_{l}^{P_{y}} \xi_{l^{\prime}}^{Q_{y}} \xi_{l}^{P_{z}} \xi_{l^{\prime}}^{Q_{z}} V\left(Q_{x} P_{x} Q_{y} P_{y} Q_{z} P_{z} \bar{r}_{f}\right) K_{D}\left[P_{x} P_{y} P_{z} \bar{r}_{f}, \tau ; \bar{r}_{i}, \hat{l}, 0\right] .
\end{aligned}
$$

Introducing the permutations $S_{i}=Q_{i} P_{i}$ with $i=x, y, z$, the quantity $\chi$ can be written as:

$$
\chi=\left(\frac{1}{2 !}\right)^{6} \sum_{P_{x} S_{x}} \sum_{P_{y} S_{y}} \sum_{P_{z} S_{z}} \sum_{l^{\prime}} \xi_{l}^{P_{x}} \xi_{l^{\prime}}^{S_{x} P_{x}^{-1}} \xi_{l}^{P_{y}} \xi_{l^{\prime}}^{S_{y} P_{y}^{-1}} \xi_{l}^{P z} \xi_{l^{\prime}}^{S_{z} P_{z}^{-1}} V\left(S_{x} S_{y} S_{z} \bar{r}_{f}\right) K_{D}\left[P_{x} P_{y} P_{z} \bar{r}_{f}, \tau ; \bar{r}_{i}, \hat{l}, 0\right] .
$$

The permutation $P_{i}$ and its inverse $P_{i}^{-1}$ have the same parity and therefore $\xi_{l^{\prime}}^{S_{i} P_{i}^{-1}}=\xi_{l^{\prime}}^{S_{i}} \xi_{l^{\prime}}^{P_{i}}$. We furthermore define $\xi_{\bar{l}}^{P_{i}} \equiv \xi_{l}^{P_{i}} \xi_{l^{\prime}}^{P_{i}}$. This leads immediately to:

$$
\chi=\sum_{\bar{l}} V_{l \bar{l}}\left(\bar{r}_{f}\right) K_{I}\left[\bar{r}_{f}, \bar{l}, \tau ; \bar{r}_{i}, \hat{l}, 0\right],
$$

where we introduced the notation: 


$$
V_{l \bar{l}}\left(\bar{r}_{f}\right)=\left(\frac{1}{2 !}\right)^{3} \sum_{S_{x}} \sum_{S_{y}} \sum_{S_{z}} \xi_{l}^{S_{x}} \xi_{l}^{S_{y}} \xi_{l}^{S_{z}} V\left(S_{x} S_{y} S_{z} \bar{r}_{f}\right) \xi_{\bar{l}}^{S_{x}} \xi_{\bar{l}}^{S_{y}} \xi_{\bar{l}}^{S_{z}}
$$

Using eq. (3) for the propagator (7) and the result obtained in (10), the evolution equation becomes:

$$
\frac{\partial}{\partial \tau} K_{I}\left[\bar{r}_{f}, l, \tau ; \bar{r}_{i}, \hat{l}, 0\right]=\frac{\hbar}{2 m} \nabla_{\bar{r}}^{2} K_{I}\left[\bar{r}_{f}, l, \tau ; \bar{r}_{i}, \hat{l}, 0\right]-\frac{1}{\hbar} \sum_{\bar{l}} V_{l \bar{l}}\left(\bar{r}_{f}\right) K_{I}\left[\bar{r}_{f}, \bar{l}, \tau ; \bar{r}_{i}, \hat{l}, 0\right],
$$

where use has been made of the invariance of $\nabla_{\bar{r}}^{2}$ with respect to permutations of the coordinates $x, y, z$ of the particle positions.

The study of the non-diagonal interaction contribution (12) is the main purpose of the present section. In some cases, these non-diagonal terms are zero. For instance, if $V(\bar{r})$ can be written as $V_{s}(\bar{r})=V(\bar{x})+V(\bar{y})+V(\bar{z})$, the invariance under the permutations of the particles implies invariance under permutations of the coordinates, which in turn then implies that $V_{l l^{\prime}}(\bar{r})=\delta_{l l^{\prime}} V(\bar{r})$. For harmonic interactions along the principal axes the many-body potential can be written as such a sum. The evolution equation is then diagonal in the quantum numbers $l$, meaning that during the evolution the boundary conditions do not change. The computational feasibility and efficiency of this type of evolution equation has been demonstrated in [1.

\section{Decomposition of the Potential}

The potential $V\left(\vec{r}_{j} ; \vec{r}_{k}\right) \equiv V\left(\vec{r}_{1}, \ldots, \vec{r}_{j}, \ldots, \vec{r}_{k}, \ldots, \vec{r}_{N}\right)$ is invariant under the permutation of any two particles, and in particular for interchanging $\vec{r}_{j}$ with $\vec{r}_{k}$

$$
V\left(\vec{r}_{j} ; \vec{r}_{k}\right)=V\left(\vec{r}_{k} ; \vec{r}_{j}\right)
$$

For motion in $2 D$, one can decompose the potential as:

$$
V\left(x_{j}, y_{j} ; x_{k}, y_{k}\right)=V_{\mathrm{bb}}\left(x_{j}, y_{j} ; x_{k}, y_{k}\right)+V_{\mathrm{ff}}\left(x_{j}, y_{j} ; x_{k}, y_{k}\right)
$$

with

$$
V_{\mathrm{bb}}\left(x_{j}, y_{j} ; x_{k}, y_{k}\right)=\frac{1}{4}\left[V\left(x_{j}, y_{j} ; x_{k}, y_{k}\right)+V\left(x_{k}, y_{j} ; x_{j}, y_{k}\right)+V\left(x_{k}, y_{k} ; x_{j}, y_{j}\right)+V\left(x_{j}, y_{k} ; x_{k}, y_{j}\right)\right]
$$

and

$$
V_{\mathrm{ff}}\left(x_{j}, y_{j} ; x_{k}, y_{k}\right)=\frac{1}{4}\left[V\left(x_{j}, y_{j} ; x_{k}, y_{k}\right)-V\left(x_{k}, y_{j} ; x_{j}, y_{k}\right)+V\left(x_{k}, y_{k} ; x_{j}, y_{j}\right)-V\left(x_{j}, y_{k} ; x_{k}, y_{j}\right)\right]
$$

Introducing the operator $\sigma_{x}, \sigma_{y}$ for interchanging the $x, y$-coordinates, and the operator $e_{x}, e_{y}$ for leaving the $x, y$ coordinates unchanged, the decomposition of the potential can be written as:

$$
V_{\mathrm{bb}}\left(x_{j}, y_{j} ; x_{k}, y_{k}\right)=\frac{1}{4}\left(e_{x}+\sigma_{x}\right)\left(e_{y}+\sigma_{y}\right) V\left(x_{j}, y_{j} ; x_{k}, y_{k}\right)
$$

For motion in $3 D$ the same principle can be used leading to the decomposition:

$$
\begin{aligned}
V\left(x_{j}, y_{j}, z_{j} ; x_{k}, y_{k}, z_{k}\right)= & V_{\mathrm{bbb}}\left(x_{j}, y_{j}, z_{j} ; x_{k}, y_{k}, z_{k}\right)+V_{\mathrm{bff}}\left(x_{j}, y_{j}, z_{j} ; x_{k}, y_{k}, z_{k}\right) \\
& +V_{\mathrm{fbf}}\left(x_{j}, y_{j}, z_{j} ; x_{k}, y_{k}, z_{k}\right)+V_{\mathrm{ffb}}\left(x_{j}, y_{j}, z_{j} ; x_{k}, y_{k}, z_{k}\right),
\end{aligned}
$$

where typically $V_{\mathrm{fbf}}\left(x_{j}, y_{j}, z_{j} ; x_{k}, y_{k}, z_{k}\right)$ is given by:

$$
V_{\mathrm{fbf}}\left(x_{j}, y_{j}, z_{j} ; x_{k}, y_{k}, z_{k}\right)=\frac{1}{8}\left(e_{x}-\sigma_{x}\right)\left(e_{y}+\sigma_{y}\right)\left(e_{z}-\sigma_{z}\right) V\left(x_{j}, y_{j}, z_{j} ; x_{k}, y_{k}, z_{k}\right)
$$

and analogous definitions hold for the other matrix elements. It is interesting to note the similarity between this projection and quaternions:

$$
1_{x y z}=\frac{1}{8}\left[\begin{array}{c}
\left(e_{x}+\sigma_{x}\right)\left(e_{y}+\sigma_{y}\right)\left(e_{z}+\sigma_{z}\right)+\left(e_{x}+\sigma_{x}\right)\left(e_{y}-\sigma_{y}\right)\left(e_{z}-\sigma_{z}\right) \\
+\left(e_{x}-\sigma_{x}\right)\left(e_{y}+\sigma_{y}\right)\left(e_{z}-\sigma_{z}\right)+\left(e_{x}-\sigma_{x}\right)\left(e_{y}-\sigma_{y}\right)\left(e_{z}+\sigma_{z}\right)
\end{array}\right] \text {. }
$$

Using this projection the formal decomposition (11) is achieved explicitly. 


\section{B. The Feynman-Kac functional}

Given that the propagator indeed satisfies the evolution equation (12), it remains to be shown that a process can be found to generate the sample paths and a Feynman-Kac functional.

\section{Positivity and the process}

To start the construction of the generator of the process consider the following identity:

$$
\begin{aligned}
\sum_{\bar{l}} V_{l \bar{l}}(\bar{r}) & =\left(\frac{1}{2 !}\right)^{3} \sum_{\bar{l}} \sum_{S_{x}} \sum_{S_{y}} \sum_{S_{z}} \xi_{l}^{S_{x}} \xi_{l}^{S_{y}} \xi_{l}^{S_{z}} V\left(S_{x} S_{y} S_{z} \bar{r}\right) \xi_{\bar{l}}^{S_{x}} \xi_{\bar{l}}^{S_{y}} \xi_{\bar{l}}^{S_{z}} \\
& =V(\bar{r})
\end{aligned}
$$

This is a direct consequence of eq. (9). Assume further, that the domain $D_{2}^{3}$ is chosen in such a way that $V_{l \bar{l}}(\bar{r}) \leq 0$ for $l \neq \bar{l}$. This allows to rewrite eq. (12) as follows:

$$
\begin{aligned}
\frac{\partial}{\partial \tau} K_{I}\left[\bar{r}_{f}, l, \tau ; \bar{r}_{i}, \hat{l}, 0\right]= & \frac{\hbar}{2 m} \nabla_{\bar{r}}^{2} K_{I}\left[\bar{r}_{f}, l, \tau ; \bar{r}_{i}, \hat{l}, 0\right]-\frac{1}{\hbar} V\left(\bar{r}_{f}\right) K_{I}\left[\bar{r}_{f}, l, \tau ; \bar{r}_{i}, \hat{l}, 0\right] \\
& -W_{l}\left(\bar{r}_{f}\right) K_{I}\left[\bar{r}_{f}, l, \tau ; \bar{r}_{i}, \hat{l}, 0\right]+\sum_{\bar{l}} W_{l \bar{l}}\left(\bar{r}_{f}\right) K_{I}\left[\bar{r}_{f}, \bar{l}, \tau ; \bar{r}_{i}, \hat{l}, 0\right]
\end{aligned}
$$

where $W_{l \bar{l}}\left(\bar{r}_{f}\right)$ denotes the non-diagonal part $-\frac{1}{\hbar} V_{l \bar{l}}\left(\bar{r}_{f}\right)$ if $l \neq \bar{l}$ :

$$
W_{l \bar{l}}\left(\bar{r}_{f}\right)=-\frac{1}{\hbar} V_{l \bar{l}}\left(\bar{r}_{f}\right)\left(1-\delta_{l, \bar{l}}\right)
$$

and the quantity $W_{l}\left(\bar{r}_{f}\right)$ stands for:

$$
W_{l}\left(\bar{r}_{f}\right)=\sum_{\bar{l}} W_{l \bar{l}}\left(\bar{r}_{f}\right)
$$

In this situation, the $W_{\bar{l}}\left(\bar{r}_{f}\right)$ can be interpreted as the rate that a walker in the position $\bar{r}_{f}$ will change its boundary condition from $l$ to $\bar{l}$. This means that the following terms of eq. (19) generate a diffusion together with a Markov process on the four possible boundary conditions:

$$
\begin{aligned}
\frac{\partial}{\partial \tau} K_{I}^{0}\left[\bar{r}_{f}, l, \tau ; \bar{r}_{i}, \hat{l}, 0\right]= & \frac{\hbar}{2 m} \nabla_{\bar{r}}^{2} K_{I}^{0}\left[\bar{r}_{f}, l, \tau ; \bar{r}_{i}, \hat{l}, 0\right]+\sum_{\bar{l}} W_{l \bar{l}}\left(\bar{r}_{f}\right) K_{I}^{0}\left[\bar{r}_{f}, \bar{l}, \tau ; \bar{r}_{i}, \hat{l}, 0\right] \\
& -W_{l}\left(\bar{r}_{f}\right) K_{I}^{0}\left[\bar{r}_{f}, l, \tau ; \bar{r}_{i}, \hat{l}, 0\right]
\end{aligned}
$$

where $K_{I}^{0}\left[\bar{r}_{f}, l, \tau ; \bar{r}_{i}, \hat{l}, 0\right]$ satisfies a backward Kolmogorov equation, and there for it is the transition probability of a compound stochastic process [10] that generates the sample paths in our state space.

\section{Positivity and the functional}

Above, we assumed that the domain $D_{2}^{3}$ can be chosen such that $V_{l \bar{l}}(\bar{r}) \leq 0$ for $l \neq \bar{l}$. This assumption is necessary, because otherwise the introduced $W$-matrix would not represent a continuous-time Markov process. From its construction it is clear that it depends on the potential. Therefore a general analysis for any potential is beyond the scope of the present communication. Assuming that the system under consideration has a potential with this property (or can be transformed by a unitary transformation to a system with such a potential), the evolution (22) describes a diffusion triggering a continuous-time Markov chain that swithces boundary conditions in a Wiener-Poisson space. The switches do not induce discontinuities in the sample paths but allow for a change in boundary condition according to the rates defined by the $W$-matrix. The sign condition on the projections of the potential is necessary for the interpretation of the non diagonal elements as rates. The evolution equation based on this assumption describes a stochastic process in the strict mathematical sense. It can therefore be simulated sign-problem-free. The propagator $K_{I}\left[\bar{r}_{f}, l, \tau ; \bar{r}_{i}, \hat{l}, 0\right]$ with the initial condition (8) can be written as a Feynman-Kac functional 


$$
K_{I}\left[\bar{r}_{f}, l, \tau ; \bar{r}_{i}, \hat{l}, 0\right]=E_{\bar{r}_{i}, \hat{l}}\left[I_{\left(\bar{R}(\tau)=\bar{r}_{f}, L_{\tau}=l\right)} \exp \left(-\frac{1}{\hbar} \sum_{\bar{l}} \int_{0}^{\tau} V_{L_{\varsigma} \bar{l}}(\bar{R}(\varsigma)) d \varsigma\right)\right],
$$

where use has been made of the identity (18) to make explicit that the potential is diagonal in the label $l$.

The sample paths are generated by a $3 N$-dimensional Brownian motion $\{\bar{R}(\tau) ; \tau \geq 0\}$ with $\sigma^{2}=\hbar \tau / m$ and absorbing and reflecting boundary conditions as defined by the index $l$. After the transition, the boundary conditions are adapted to agree with those from the Markov chain $\left\{L_{\tau} ; \tau \geq 0\right\}$ where the (normalized) quantities $V_{l \bar{l}}$ then determine the boundary-condition transition rates $\bar{l} \rightarrow l$. Repeated application of this procedure for all the subprocesses starting with boundary conditions $\bar{l}$ generates the sample paths of the diffusion process of $N-2$ distinguishable and two indistinguishable particles in three dimensions. The stochastic integration over the Feynman-Kac functional is then done by killing and branching.

\section{DIFFUSION ON A REDUCED STATE SPACE $\widehat{D}$}

The positivity of the rates $W_{l \bar{l}}\left(\bar{r}_{f}\right)$ is a necessary ingredient to guarantee the interpretation of the Euclidean time Schrödinger equation as a compound diffusion equation with killing and branching in a Wiener-Poisson space. For some potentials, the condition of negative definite $V_{l \bar{l}}\left(\bar{r}_{f}\right)$ may not be satisfied on the state space $D_{2}^{3} \otimes \mathbb{R}^{3(N-2)}$. In these cases, the definition of an alternative (reduced) state space is required in order to find purely positive transition rates $W_{l \bar{l}}\left(\bar{r}_{f}\right)$. The symmetry of the potential effects the structure of the adapted state space, which therewith is specific for certain classes of applications. To illustrate the construction principle, the reduced state space $\widehat{D}_{2}^{3} \otimes \mathbb{R}^{3(N-2)}$ will be considered, where

$$
\widehat{D}_{2}^{3} \equiv\left\{\left(x_{j}, x_{k}, y_{j}, y_{k}, z_{j}, z_{k}\right)^{T}\left|x_{j} \geq\right| x_{k}\left|, y_{j} \geq\right| y_{k}\left|, z_{j} \geq\right| z_{k} \mid\right\}
$$

This choice will be of relevance for the probabilistic sampling of the real ground-state wave function of ortho-helium. The procedures for the construction of an ordered state space $x_{j} \geq x_{k}, y_{j} \geq y_{k}, z_{j} \geq z_{k}$, reported in 2 近, alone do not induce the ordering on $\widehat{D}_{2}^{3}$, and an additional set of boundary conditions must be supplied to impose the desired ordering. In that framework, the separation of the multi-dimensional diffusion process into one-dimensional diffusion processes will again prove useful.

\section{A. Free Diffusion of Indistinguishable Particles on $\widehat{D}_{2}^{1}$}

The free diffusion process of two indistinguishable particles on a line has been introduced in [2] as the diffusion process of two distinguishable particles on the ordered state space $D_{2}^{1}$ with absorption (for fermions) or reflection (for bosons) at the boundary $\partial D_{2}^{1}$. The construction of the free diffusion process on the reduced state space $\widehat{D}_{2}^{1}$ requires the extension of the stochastic process. (Anti)symmetrization again plays the major role in that approach: the diffusion process of free indistinguishable particles on the state space $D_{2}^{1}$ can be mapped onto $\widehat{D}_{2}^{1}$ by the construction of reflecting and absorbing boundaries. The starting point for the derivation of the required processes is the separation of the state space into two non-intersecting subspaces $\widehat{D}_{2}^{1}$ and $\widehat{P}_{x} \widehat{D}_{2}^{1}$ of equal volume: $D_{2}^{1}=\widehat{D}_{2}^{1} \cup \widehat{P}_{x} \widehat{D}_{2}^{1}$. The mapping between both subspaces is realized by the permutation operator $\widehat{P}_{x}$ which models reflection at $x_{1}=-x_{2}$, i.e.: $\widehat{P}_{x}\left(x_{1}, x_{2}\right)^{T}=\left(-x_{2},-x_{1}\right)^{T}$. Then, the transition probability density $\rho_{\mathrm{f}, \mathrm{b}}\left(\bar{x}, \tau ; \bar{x}^{\prime}\right)$ that the system of two free fermions/bosons evolves from $\bar{x}^{\prime}$ to $\bar{x}$ during the Euclidean time interval $\tau$, can be written as

$$
\rho_{\mathrm{f}, \mathrm{b}}\left(\bar{x}, \tau ; \bar{x}^{\prime}\right)=\frac{1}{2}\left(\rho_{\mathrm{f}, \mathrm{b}}^{\mathrm{f}}\left(\bar{x}, \tau ; \bar{x}^{\prime}\right)+\rho_{\mathrm{f}, \mathrm{b}}^{\mathrm{b}}\left(\bar{x}, \tau ; \bar{x}^{\prime}\right)\right),
$$

where we defined

$$
\rho_{\mathrm{f}, \mathrm{b}}^{\mathrm{f}}\left(\bar{x}, \tau ; \bar{x}^{\prime}\right)=\rho_{\mathrm{f}, \mathrm{b}}\left(\bar{x}, \tau ; \bar{x}^{\prime}\right)-\rho_{\mathrm{f}, \mathrm{b}}\left(\widehat{P}_{x} \bar{x}, \tau ; \bar{x}^{\prime}\right) \quad, \quad \rho_{\mathrm{f}, \mathrm{b}}^{\mathrm{b}}\left(\bar{x}, \tau ; \bar{x}^{\prime}\right)=\rho_{\mathrm{f}, \mathrm{b}}\left(\bar{x}, \tau ; \bar{x}^{\prime}\right)+\rho_{\mathrm{f}, \mathrm{b}}\left(\widehat{P}_{x} \bar{x}, \tau ; \bar{x}^{\prime}\right) .
$$

Each of the transition probability densities $\rho_{\mathrm{f}, \mathrm{b}}\left(\bar{x}, \tau ; \bar{x}^{\prime}\right)$ consists of two density matrices, one $-\rho_{\mathrm{f}, \mathrm{b}}^{\mathrm{b}}\left(\bar{x}, \tau ; \bar{x}^{\prime}\right)$ - for which the boundary $x_{1}=-x_{2}$ acts reflecting, and one $-\rho_{\mathrm{f}, \mathrm{b}}^{\mathrm{f}}\left(\bar{x}, \tau ; \bar{x}^{\prime}\right)$-which induces absorption at $x_{1}=-x_{2}$. With an appropriate initial choice $\bar{x}^{\prime} \in \widehat{D}_{2}^{1}$, free fermion/boson diffusion to any point $\bar{x} \in D_{2}^{1}$-and thus also to any 
$\bar{x} \in \mathbb{R}^{2}$ - can be projected to the reduced state space $\widehat{D}_{2}^{1}$ by means of reflecting and absorbing state-space boundaries. Each of the elementary density matrices $\rho_{\mathrm{f}, \mathrm{b}}^{\mathrm{b}}\left(\bar{x}, \tau ; \bar{x}^{\prime}\right)$ and $\rho_{\mathrm{f}, \mathrm{b}}^{\mathrm{f}}\left(\bar{x}, \tau ; \bar{x}^{\prime}\right)$ constitutes a diffusion process with the initial condition

$$
\lim _{\tau \downarrow 0} \rho_{\mathrm{f}, \mathrm{b}}^{\mathrm{b}}\left(\bar{x}, \tau ; \bar{x}^{\prime}\right)=\lim _{\tau \downarrow 0} \rho_{\mathrm{f}, \mathrm{b}}^{\mathrm{f}}\left(\bar{x}, \tau ; \bar{x}^{\prime}\right)=\delta\left(\bar{x}-\bar{x}^{\prime}\right)
$$

To proof this statement, $\rho_{\mathrm{f}}^{\mathrm{b}}\left(\bar{x}, \tau ; \bar{x}^{\prime}\right)$ and $\rho_{\mathrm{f}}^{\mathrm{f}}\left(\bar{x}, \tau ; \bar{x}^{\prime}\right)$ must be shown to satisfy probability conservation and the semigroup property. Both properties are derived taking into account the Markov properties for the free fermion/boson diffusion density $\rho_{\mathrm{f}, \mathrm{b}}\left(\bar{x}, \tau ; \bar{x}^{\prime}\right)$ defined on the state space $D_{2}^{1}$ with absorbing/reflecting boundaries. Probability conservation for the two-boson density $\rho_{\mathrm{b}}^{\mathrm{b}}\left(\bar{x}, \tau ; \bar{x}^{\prime}\right)$ follows from

$$
\int_{\widehat{D}_{2}^{1}} d \bar{x} \rho_{\mathrm{b}}^{\mathrm{b}}\left(\bar{x}, \tau ; \bar{x}^{\prime}\right)=\frac{1}{2} \int_{\widehat{D}_{2}^{1}} d \bar{x} \sum_{\widehat{P}_{x}} \rho_{\mathrm{b}}^{\mathrm{b}}\left(\widehat{P}_{x} \bar{x}, \tau ; \bar{x}^{\prime}\right)=\frac{1}{2} \int_{D_{2}^{1}} d \bar{x} \rho_{\mathrm{b}}^{\mathrm{b}}\left(\bar{x}, \tau ; \bar{x}^{\prime}\right)=\frac{1}{2} \int_{D_{2}^{1}} d \bar{x}\left(\rho_{\mathrm{b}}\left(\bar{x}, \tau ; \bar{x}^{\prime}\right)+\rho_{\mathrm{b}}\left(\widehat{P}_{x} \bar{x}, \tau ; \bar{x}^{\prime}\right)\right)=1 .
$$

As argued in [2], the free fermion diffusion density $\rho_{\mathrm{f}}\left(\bar{x}, \tau ; \bar{x}^{\prime}\right)$ is not automatically normalized on the state space $D_{2}^{1}$. In contrast, normalization of the free fermion diffusion density $\rho_{\mathrm{f}}\left(\bar{x}, \tau ; \bar{x}^{\prime}\right)$ requires the introduction of the absorbing boundary state [2] which comprises all transitions to the outside of the state space $D_{2}^{1}$. Also in the present analysis, the construction of a boundary state [8] [11] ensures the normalization of the probability densities $\rho_{\mathrm{b}}^{\mathrm{f}}\left(\bar{x}, \tau ; \bar{x}^{\prime}\right), \rho_{\mathrm{f}}^{\mathrm{b}}\left(\bar{x}, \tau ; \bar{x}^{\prime}\right)$ and $\rho_{\mathrm{f}}^{\mathrm{f}}\left(\bar{x}, \tau ; \bar{x}^{\prime}\right)$. The density $\rho_{\mathrm{b}}^{\mathrm{f}}\left(\bar{x}, \tau ; \bar{x}^{\prime}\right)$, for instance, associates an absorbing boundary $x_{1}=-x_{2}$ to bear in mind the probability for distinguishable-particle propagation to $x_{1} \leq-x_{2}$. The semigroup properties of $\rho_{\mathrm{f}}^{\mathrm{f}}\left(\bar{x}, \tau ; \bar{x}^{\prime}\right), \rho_{\mathrm{f}}^{\mathrm{b}}\left(\bar{x}, \tau ; \bar{x}^{\prime}\right)$, $\rho_{\mathrm{b}}^{\mathrm{f}}\left(\bar{x}, \tau ; \bar{x}^{\prime}\right)$ and $\rho_{\mathrm{b}}^{\mathrm{b}}\left(\bar{x}, \tau ; \bar{x}^{\prime}\right)$ are derived by unfolding to the state space $D_{2}^{1}$. Using their symmetry properties, they can be retraced to the semigroup properties of $\rho_{\mathrm{f}}\left(\bar{x}, \tau ; \bar{x}^{\prime}\right)$ and $\rho_{\mathrm{b}}\left(\bar{x}, \tau ; \bar{x}^{\prime}\right)$. E.g.

$$
\begin{aligned}
\int_{\widehat{D}_{2}^{1}} d \bar{x} \rho_{\mathrm{f}}^{\mathrm{f}}\left(\bar{x}^{\prime}, \tau ; \bar{x}\right) \rho_{\mathrm{f}}^{\mathrm{f}}\left(\bar{x}, \sigma ; \bar{x}^{\prime \prime}\right) & =\frac{1}{2} \int_{\widehat{D}_{2}^{1}} d \bar{x} \sum_{\widehat{P}_{x}} \rho_{\mathrm{f}}^{\mathrm{f}}\left(\bar{x}^{\prime}, \tau ; \widehat{P}_{x} \bar{x}\right) \rho_{\mathrm{f}}^{\mathrm{f}}\left(\widehat{P}_{x} \bar{x}, \sigma ; \bar{x}^{\prime \prime}\right) \\
& =\frac{1}{2} \int_{D_{2}^{1}} d \bar{x}\left(\rho_{\mathrm{f}}\left(\bar{x}^{\prime}, \tau ; \bar{x}\right)-\rho_{\mathrm{f}}\left(\bar{x}^{\prime}, \tau ; \widehat{P}_{x} \bar{x}\right)\right)\left(\rho_{\mathrm{f}}\left(\bar{x}, \sigma ; \bar{x}^{\prime \prime}\right)-\rho_{\mathrm{f}}\left(\widehat{P}_{x} \bar{x}, \sigma ; \bar{x}^{\prime \prime}\right)\right) \\
& =\int_{D_{2}^{1}} d \bar{x}\left(\rho_{\mathrm{f}}\left(\bar{x}^{\prime}, \tau ; \bar{x}\right) \rho_{\mathrm{f}}\left(\bar{x}, \sigma ; \bar{x}^{\prime \prime}\right)-\rho_{\mathrm{f}}\left(\bar{x}^{\prime}, \tau ; \bar{x}\right) \rho_{\mathrm{f}}\left(\bar{x}, \sigma ; \widehat{P}_{x} \bar{x}^{\prime \prime}\right)\right) \\
& =\rho_{\mathrm{f}}\left(\bar{x}^{\prime}, \tau+\sigma ; \bar{x}^{\prime \prime}\right)-\rho_{\mathrm{f}}\left(\bar{x}^{\prime}, \tau+\sigma ; \widehat{P}_{x} \bar{x}^{\prime \prime}\right)=\rho_{\mathrm{f}}^{\mathrm{f}}\left(\bar{x}^{\prime}, \tau+\sigma ; \bar{x}^{\prime \prime}\right) .
\end{aligned}
$$

As a consequence, the free diffusion process $\widehat{X}_{\mathrm{f}, \mathrm{b}}(\tau)$ of two indistinguishable particles on a line is characterized by the combination of two adapted Wiener processes defined on the reduced state space $\widehat{D}_{2}^{1}$ :

$$
\begin{aligned}
\widehat{X}_{\mathrm{b}}(\tau) & =\frac{1}{2}\left(\widehat{X}_{\mathrm{b}}^{\mathrm{b}}(\tau)+\widehat{X}_{\mathrm{b}}^{\mathrm{f}}(\tau)\right) \text { for bosons, and } \\
\widehat{X}_{\mathrm{f}}(\tau) & =\frac{1}{2}\left(\widehat{X}_{\mathrm{f}}^{\mathrm{b}}(\tau)+\widehat{X}_{\mathrm{f}}^{\mathrm{f}}(\tau)\right) \text { for fermions }
\end{aligned}
$$

Since $\rho_{\mathrm{b}, \mathrm{f}}^{\mathrm{b}}\left(\bar{x}, \tau ; \bar{x}^{\prime}\right)$ and $\rho_{\mathrm{b}, \mathrm{f}}^{\mathrm{f}}\left(\bar{x}, \tau ; \bar{x}^{\prime}\right)$ are orthogonal, e.g.

$$
\begin{aligned}
\int_{D_{2}^{1}} d \bar{x} \rho_{\mathrm{f}}^{\mathrm{f}}\left(\bar{x}^{\prime}, \tau ; \bar{x}\right) \rho_{\mathrm{f}}^{\mathrm{b}}\left(\bar{x}, \tau ; \bar{x}^{\prime \prime}\right) & =\int_{\widehat{D}_{2}^{1}} d \bar{x} \rho_{\mathrm{f}}^{\mathrm{f}}\left(\bar{x}^{\prime}, \tau ; \bar{x}\right) \rho_{\mathrm{f}}^{\mathrm{b}}\left(\bar{x}, \tau ; \bar{x}^{\prime \prime}\right)+\int_{\widehat{P}_{x} \widehat{D}_{2}^{1}} d \bar{x} \rho_{\mathrm{f}}^{\mathrm{f}}\left(\bar{x}^{\prime}, \tau ; \bar{x}\right) \rho_{\mathrm{f}}^{\mathrm{b}}\left(\bar{x}, \tau ; \bar{x}^{\prime \prime}\right) \\
& =\int_{\widehat{D}_{2}^{1}} d \bar{x}\left[\rho_{\mathrm{f}}^{\mathrm{f}}\left(\bar{x}^{\prime}, \tau ; \bar{x}\right) \rho_{\mathrm{f}}^{\mathrm{b}}\left(\bar{x}, \tau ; \bar{x}^{\prime \prime}\right)-\rho_{\mathrm{f}}^{\mathrm{f}}\left(\bar{x}^{\prime}, \tau ; \bar{x}\right) \rho_{\mathrm{f}}^{\mathrm{b}}\left(\bar{x}, \tau ; \bar{x}^{\prime \prime}\right)\right]=0
\end{aligned}
$$

each of the densities $\rho_{\mathrm{b}, \mathrm{f}}^{\mathrm{b}}\left(\bar{x}, \tau ; \bar{x}^{\prime}\right), \rho_{\mathrm{b}, \mathrm{f}}^{\mathrm{f}}\left(\bar{x}, \tau ; \bar{x}^{\prime}\right)$ evolves independently and generates the corresponding Brownian motion. We have used in the upper and the lower indices the same letter to characterize the boundary condition, this is customarily done in supersymmetric models [12], it should be noted that these letters for b in the upper indices indicate wether there is absorption or reflection on the boundary introduced by the (broken) symmetry requirements for the potential and they are independent of the $\mathrm{f}$ or $\mathrm{b}$ in the under indices introduced by the statistics of the particles.

In the sampling procedures, each of the processes $\widehat{X}_{\mathrm{f}, \mathrm{b}}^{\mathrm{f} b}(\tau)$ is constructed from the diffusion process $X_{\mathrm{d}}(\tau)$ of two distinguishable particles taking into account the corresponding boundary conditions: the process $\widehat{X}_{\mathrm{b}}^{\mathrm{f}}(\tau)$, for instance, means reflection at the hypersurface $x_{1}=x_{2}$ and absorption at $x_{1}=-x_{2}$. For each of the composite processes, 


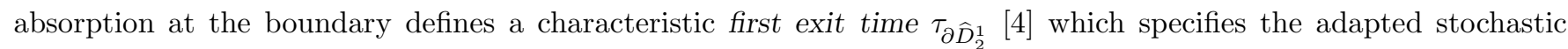
process, e.g.

$$
\widehat{X}_{\mathrm{f}}^{\mathrm{f}}(\tau)=\left\{\begin{array}{ll}
X_{\mathrm{d}}(\tau) & \text { for } \tau \leq \tau_{\partial \widehat{D}_{2}^{1}} \\
X_{\mathrm{d}}\left(\tau_{\partial \widehat{D}_{2}^{1}}\right) & \text { for } \tau>\tau_{\partial \widehat{D}_{2}^{1}}
\end{array} .\right.
$$

In fig. 1, the feasibility of the developed sampling technique is indicated by graphical comparison of the numerically sampled and rigorous transition probability densities $\rho_{\mathrm{f}}^{\mathrm{b}}$ for a given initial $\bar{x}^{\prime}$ denoted by the crosses.

\section{B. Free Fermion and Free Boson Diffusion on $\widehat{D}_{2}^{3}$}

We now formulate the diffusion process of two free indistinguishable particles in three dimensions on the reduced state space $\widehat{D}_{2}^{3}$ by extending the symmetry analysis performed above. Following the basic principles outlined in $\left[\begin{array}{|l}\text {, } \\ \text {, }\end{array}\right.$ the free diffusion of indistinguishable particles can be traced back to one-dimensional free diffusion processes. In that framework, the transition probabilities $X_{\mathrm{f}, \mathrm{b}}^{\mathrm{f}, \mathrm{b}}(\tau)$ represent the elementary subprocesses combined to constitute the overall free diffusion process in three dimensions. For free identical fermions in three dimensions, the density matrix can be expressed as

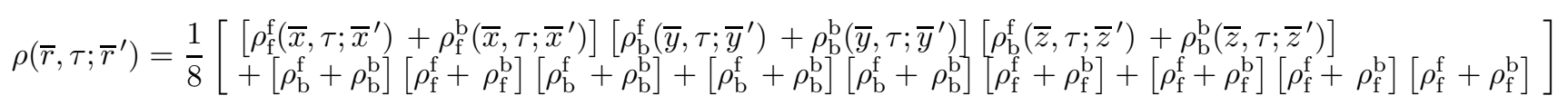

whereas for the corresponding boson system the density matrix evaluates to

$$
\rho\left(\bar{r}, \tau ; \bar{r}^{\prime}\right)=\frac{1}{8}\left[\begin{array}{c}
{\left[\rho_{\mathrm{b}}^{\mathrm{f}}\left(\bar{x}, \tau ; \bar{x}^{\prime}\right)+\rho_{\mathrm{b}}^{\mathrm{b}}\left(\bar{x}, \tau ; \bar{x}^{\prime}\right)\right]\left[\rho_{\mathrm{b}}^{\mathrm{f}}\left(\bar{y}, \tau ; \bar{y}^{\prime}\right)+\rho_{\mathrm{b}}^{\mathrm{b}}\left(\bar{y}, \tau ; \bar{y}^{\prime}\right)\right]\left[\begin{array}{c}
\left.\rho_{\mathrm{b}}^{\mathrm{f}}\left(\bar{z}, \tau ; \bar{z}^{\prime}\right)+\rho_{\mathrm{b}}^{\mathrm{b}}\left(\bar{z}, \tau ; \bar{z}^{\prime}\right)\right] \\
+\left[\rho_{\mathrm{f}}^{\mathrm{f}}+\rho_{\mathrm{f}}^{\mathrm{b}}\right]\left[\rho_{\mathrm{f}}^{\mathrm{f}}+\rho_{\mathrm{f}}^{\mathrm{b}}\right]\left[\rho_{\mathrm{b}}^{\mathrm{f}}+\rho_{\mathrm{b}}^{\mathrm{b}}\right]+\left[\rho_{\mathrm{b}}^{\mathrm{f}}+\rho_{\mathrm{b}}^{\mathrm{b}}\right]\left[\rho_{\mathrm{f}}^{\mathrm{f}}+\rho_{\mathrm{f}}^{\mathrm{b}}\right]\left[\rho_{\mathrm{f}}^{\mathrm{f}}+\rho_{\mathrm{f}}^{\mathrm{b}}\right]+\left[\rho_{\mathrm{f}}^{\mathrm{f}}+\rho_{\mathrm{f}}^{\mathrm{b}}\right]\left[\rho_{\mathrm{b}}^{\mathrm{f}}+\rho_{\mathrm{b}}^{\mathrm{b}}\right]\left[\rho_{\mathrm{f}}^{\mathrm{f}}+\rho_{\mathrm{f}}^{\mathrm{b}}\right]
\end{array}\right]}
\end{array}\right.
$$

In this representation, the density matrix for identical particles contains 32 terms. Since each term factorizes into a product of three one-dimensional orthogonal density matrices, each of the 32 elementary three-dimensional density matrices satisfies the Markov properties and generates a Wiener subprocess on the reduced state space $\widehat{D}_{2}^{3}$. For instance, the subprocess $\widehat{X}_{\mathrm{fbb}}^{\mathrm{fbf}}(\tau)$

$$
\widehat{X}_{\mathrm{fbb}}^{\mathrm{fbf}}(\tau)=\widehat{X}_{\mathrm{f}}^{\mathrm{f}}(\tau) \otimes \widehat{Y}_{\mathrm{b}}^{\mathrm{b}}(\tau) \otimes \widehat{Z}_{\mathrm{b}}^{\mathrm{f}}(\tau),
$$

can be sampled as the diffusion process of two free distinguishable particles restricted to the reduced state space by reflection at the boundaries $y_{1}=y_{2}$ and $z_{1}=z_{2}$ and absorption at $x_{1}=x_{2}, x_{1}=-x_{2}, y_{1}=-y_{2}$ and $z_{1}=-z_{2}$. The combination of all the 64 Wiener subprocesses gives rise to the free diffusion process of two indistinguishable particles in three dimensions defined on $\widehat{D}_{2}^{3}$. The symmetry properties of the elementary processes allow furthermore to unfold the resulting probability density into the configuration space $\mathbb{R}^{6}$.

\section{Evolution Equations and Decomposition of the Potential}

The derivation of the evolution equation for the propagator proceeds along the same lines as for the two-dimensional case, as discussed in Sec. II.A.2. Without going into detail, we only mention the result

$$
\frac{\partial}{\partial \tau} K_{I}\left[\bar{r}_{f}, l, l^{\prime}, \tau ; \bar{r}_{i}, \hat{l}, \hat{l}^{\prime}, 0\right]=\frac{\hbar}{2 m} \nabla_{\bar{r}}^{2} K_{I}\left[\bar{r}_{f}, l, l^{\prime}, \tau ; \bar{r}_{i}, \hat{l}, \hat{l}^{\prime}, 0\right]-\frac{1}{\hbar} \sum_{\bar{l}, \bar{l}^{\prime}} V_{l \bar{l}}^{l^{\prime} \bar{l}^{\prime}}\left(\bar{r}_{f}\right) K_{I}\left[\bar{r}_{f}, \bar{l}, \bar{l}^{\prime}, \tau ; \bar{r}_{i}, \hat{l}, \hat{l}^{\prime}, 0\right]
$$

where

$$
K_{I}\left[\bar{r}_{f}, l, l^{\prime}, \tau ; \bar{r}_{i}, \hat{l}, \hat{l}^{\prime}, 0\right]=\left(\frac{1}{2 !}\right)^{6} \sum_{\widehat{P}_{x}} \xi_{l^{\prime}} \widehat{P}_{x} \sum_{\widehat{P}_{y}} \xi_{l^{\prime}}^{\widehat{P}_{y}} \sum_{\widehat{P}_{z}} \xi_{l^{\prime}} \widehat{P}_{z} \sum_{P_{x}} \xi_{l}^{P_{x}} \sum_{P_{y}} \xi_{l}^{P_{y}} \sum_{P_{z}} \xi_{l}^{P_{z}} K_{D}\left[\widehat{P}_{x} \widehat{P}_{y} \widehat{P}_{z} P_{x} P_{y} P_{z} \bar{r}_{f}, \tau ; \bar{r}_{i}, \hat{l}, 0\right]
$$

The components of the potential are given by

$$
V_{l \bar{l}}^{l^{\prime} \bar{l}^{\prime}}\left(\bar{r}_{f}\right)=\left(\frac{1}{2 !}\right)^{6} \sum_{\widehat{S}_{x}} \sum_{\widehat{S}_{y}} \sum_{\widehat{S}_{z}} \sum_{S_{x}} \sum_{S_{y}} \sum_{S_{z}} \xi_{l}^{\widehat{S}_{x}} \xi_{l}^{\widehat{S}_{y}} \xi_{l}^{\widehat{S}_{z}} \xi_{l^{\prime}}^{S_{x}} \xi_{l^{\prime}}^{S_{y}} \xi_{l^{\prime}}^{S_{z}} V\left(\widehat{S}_{x} \widehat{S}_{y} \widehat{S}_{z} S_{x} S_{y} S_{z} \bar{r}_{f}\right) \xi_{\bar{l}}^{S_{x}} \xi_{\bar{l}}^{S_{y}} \xi_{\bar{l}}^{S_{z}} \xi_{\bar{l}^{\prime}}^{\widehat{S}_{x}} \xi_{\bar{l}^{\prime}}^{\widehat{S}_{y}} \xi_{\bar{l}^{\prime}}^{\widehat{S}_{z}}
$$

it should be noted that if the range of the label $l$ used to denoted the statistics is extented in such a way that it also indicates the boundary conditions on the reduced domain then the evolution equation for the propagator derived here takes the same form as the equation (19). 


\section{AN ALGORITHMIC APPROACH ON MODELS}

In the previous section, we introduced a novel representation of the Euclidean-time propagator of systems containing two indistinguishable particles. This representation allows to sample the propagator sign-problem-free. We now develop a numerically feasible sign-problem-free implementation scheme. Its efficiency in numerical practice is analyzed in applications to model systems. In all the cases, we use atomic units $\hbar=m=e=1$. Energy and Euclidean time are measured in Hartrees $(\mathrm{H})$ and $\mathrm{H}^{-1}$.

\section{A. General Structure}

The key elements of the many-body diffusion algorithm (MBDA) for the symmetry problems considered here are (i) an appropriately adapted free diffusion step, (ii) a routine to model subprocess transitions, and (iii) a branching and killing procedure. Whilst some elements of the algorithm resemble Diffusion Monte Carlo, we stress that the underlying principle of interdependent subprocess evolution is conceptually new. We are also aware that the efficiency of the presented algorithm can be drastically enhanced by importance sampling methods. Although we can show that this expectation holds true, we do not dwell on these refinements, for major emphasis is on the realization of symmetry arguments. In the limit of infinitely long evolution $\tau \rightarrow \infty$, the stepwise application of the Euclidean time propagator on a given sample leads in principle to the system's lowest eigenstate compatible with the symmetry restrictions made. In numerical practice, this evolution occurs in discrete Euclidean time steps $\Delta \tau$. Since the assumption of such time steps is only exact for $\Delta \tau \rightarrow 0$, the algorithm in principle suffers from a systematic time-step error. The latter, however, could be controlled in the considered applications. The main advantages of the developed approach are the simplicity and convergence properties. In particular, it allows for the transparent implementation of reflecting and absorbing boundaries to model the subprocesses derived above. Let us discuss the algorithm in some more detail. Having generated an initial population of walkers located at positions $\left(\bar{r}_{i}^{\prime}\right)_{i}$ in the appropriate state space, each valid walker makes a free distinguishable particle move to $\left(\tilde{r}_{f}^{\prime}\right)_{i}$. This is achieved by the construction of Gaussian deviates with variance $\sqrt{\Delta \tau}$ and mean $\bar{r}_{i}^{\prime}$. If a walker hits the state-space boundary by free distinguishable particle diffusion it is either reflected or absorbed depending on its characteristic boundary conditions [1]. In numerical practice, the use of non-zero Euclidean time steps $\Delta \tau$ prevents the proper construction of reflecting and absorbing boundaries. In the mathematical literature, free diffusion in the presence of an absorbing boundary is realized by the so-called Skohorod construction [8]. The latter reflects walker trajectories without changing the "momentum" of the walker. This is in contrast to the reflection experienced by a classical object at a hard wall. In the numerical procedures, the final reflected position $\bar{r}_{f}^{\prime}$ is obtained by reflection of the corresponding $\tilde{r}_{f}^{\prime}$. The systematic error for inappropriate reflection vanishes for $\Delta \tau \rightarrow 0$; for the utilized time intervals $\Delta \tau$ no significant error were observed. The efficient implementation of fermionlike diffusion, in contrast, calls for numerical crossing-recrossing corrections. For two identical fermions $j, k$ on a line the corrections for an absorbing boundary $x_{j}=x_{k}$ have been discussed in [1]. Based on the principle of images, we showed that an apparently valid walker move from $\left(x_{j}^{\prime}, x_{k}^{\prime}\right)$ to $\left(x_{j}, x_{k}\right)$, both points inside the state space, has to be rejected with the probability $\exp \left(-\left(x_{j}-x_{k}\right)\left(x_{j}^{\prime}-x_{k}^{\prime}\right) / \Delta \tau\right)$. The symmetry properties of the potentials discussed in this work require the generation of interdependent subprocesses. In succession to the adapted free diffusion step, a walker associated with the boundary condition of the subprocess $l$ may change to the boundary condition of a subprocess $l^{\prime}$ with the probability $p_{l \rightarrow l^{\prime}}\left(\bar{r}_{f}\right)$. Its actual structure is determined by the physical situation, e.g. the symmetry properties of both the potential and the eigenstate under consideration. The local dependence of the probabilities $p_{l \rightarrow l^{\prime}}\left(\bar{r}_{f}\right)$ governs the relevance of subprocess transitions at a given position $\bar{r}_{f}$. The structure of $p_{l \rightarrow l^{\prime}}\left(\bar{r}_{f}\right)$ is fairly flexible as long as it harmonizes with the killing and branching procedure. For instance, $p_{l \rightarrow l^{\prime}}\left(\bar{r}_{f}\right)$ could be decoupled from the different kinds of boundary conditions. Then each subprocess transition occurs with equal probability $1 / n_{l}$, where $n_{l}$ denotes the total number of subprocesses. Accordingly, both the normalization rates and the probability for branching and killing become dependent on the subprocess transition performed on the walker. In what follows, we assume subprocess transition probabilities adapted to the branching and killing procedure due to the potential. The third step, branching and killing, realizes the presence of the potential by the implementation

of the exponential path-integral weights $w\left(\bar{r}^{\prime}, \bar{r}\right)=\left.\exp \left(-\int_{0}^{\Delta \tau} V(\bar{R}(\varsigma)) d \varsigma\right)\right|_{\bar{R}(0)=\bar{r}^{\prime}} ^{\bar{R}(\Delta \tau)=\bar{r}}$, where $\bar{R}(\varsigma)$ denotes the paths generated by the adapted free diffusion process. The principal lack of continuous trajectories in discrete time-step evolution procedures induces the necessity for feasible approximations to $w\left(\bar{r}^{\prime}, \bar{r}\right)$. For sufficiently smooth potentials, the Suzuki-Trotter formula [6] offers a reliable alternative. Then only the values of the potential at the initial and final points of a move are taken into account: $w\left(\bar{r}^{\prime}, \bar{r}\right)=\exp \left\{-\left(V\left(\bar{r}^{\prime}\right)+V(\bar{r})\right) \Delta \tau / 2\right\}$. Especially for singular potentials, improved approximations (for a detailed discussion see e.g. [7] and references therein) are recommended. Here, we 
choose the semiclassical approximation suggested in [9]: $w\left(\bar{r}^{\prime}, \bar{r}\right)=\exp \left\{-\int_{0}^{1} d \xi V\left(\bar{r}^{\prime}+\xi\left(\bar{r}-\bar{r}^{\prime}\right)\right)\right\}$. A branching and killing technique serves to efficiently adapt the walkers to the weights $w\left(\bar{r}^{\prime}, \bar{r}\right)$ by an acceptance/rejection procedure. To conserve the size of the walker population to within statistical fluctuations, an appropriate reference energy $E_{\text {ref }}$ has been supplied to yield improved weights $w\left(\bar{r}^{\prime}, \bar{r}\right) e^{E_{\text {ref }} \Delta \tau}$. In [1], we derived an estimator for the ground-state energy $E_{0}$. The key elements of this estimator are the potential average $\langle V\rangle_{D}$ over the state space $D$ and a surface term $\langle j\rangle_{\partial D}$ triggered by the non-zero gradient of the fermionlike subdensities at absorbing boundaries. The estimate is easily generalized to an ensemble of interdependent subdensities $\Psi(\bar{r}, l, \tau)$

$$
E_{0}=\lim _{\tau \rightarrow \infty} E_{\tau}=\lim _{\tau \rightarrow \infty} \sum_{l}\left[-\frac{\frac{\hbar^{2}}{2 m} \int_{\partial D_{2}} d \bar{r} \bar{\nabla}_{\bar{r}} \Psi(\bar{r}, l, \tau)}{\int_{D_{2}} d \bar{r} \Psi(\bar{r}, l, \tau)}+\frac{\int_{D_{2}} d \bar{r} \Psi(\bar{r}, l, \tau) V(\bar{r})}{\int_{D_{2}} d \bar{r} \Psi(\bar{r}, l, \tau)}\right]=\sum_{l}\left[\langle j\rangle_{\partial D_{2}}^{l}+\langle V\rangle_{D_{2}}^{l}\right] .
$$

Alternatively, the growth estimates

$$
E_{0}=\lim _{\tau \rightarrow \infty} E_{\tau}=-\lim _{\tau \rightarrow \infty} \frac{1}{\tau} \ln \int d \bar{r}_{f} \int d \bar{r}_{i} \sum_{l} K\left[\bar{r}_{f}, l, \tau ; \bar{r}_{i}, 0\right],
$$

may serve to predict the ground-state energy. Based on this general algorithmic structure, the ground-state wave function of several model systems is investigated below. The different types of potentials make a specific analysis of the appropriate state space necessary.

\section{B. Double well and Mexican Hat}

To start with, the symmetry principles underlying the many-body diffusion algorithm are illustrated for a quantummechanical particle in a double-well or a Mexican Hat potential. For these models, the utilization of (anti)symmetric subprocesses is shown to provide a suitable technique for the numerical generation of the real ground-state wave function. Our study aims at revealing the role of symmetry arguments to sample a probability density on an appropriate part of the configuration space. Feasibility of the symmetry concept has been tested independently with standard Monte Carlo techniques. Consider a single particle in a one-dimensional double-well potential, described by the Hamiltonian

$$
H=-\frac{1}{2} \frac{\partial^{2}}{\partial x^{2}}+V(x) \quad \text { with } \quad V(x)=\frac{\left(x^{2}-a\right)^{2}-a^{2}}{4}+b x^{3},
$$

where $a$ and $b$ represent parameters. Fig. 2 depicts the potential values for two different parameter choices: $a=2, b=0$ and $a=2, b=0.25$ in the following referred to as symmetric and asymmetric potential. According to the rules outlined above, the Euclidean time propagator $K\left(x, \tau ; x^{\prime}\right)$ associated with the Hamiltonian (27) can be formulated as a sum of four subpropagators $K\left(x, \tau, \jmath ; x^{\prime}, \tilde{\jmath}\right)$ with $\jmath, \tilde{\jmath} \in\{0,1\}$ denoting symmetric resp. antisymmetric behavior under the transformation $\bar{P}_{x}: x \rightarrow-x$. This means that $K\left(x, \jmath, \tau ; x^{\prime}, 0\right)$ and $K\left(x, \jmath, \tau ; x^{\prime}, 1\right)$ are initially symmetric and antisymmetric with respect to the boundary $x=0$ which separates the two half-spaces $D^{-}=\{x \mid x \leq 0\}$ and $D^{+}=\{x \mid x \geq 0\}$. The (anti)symmetrized potentials $V_{\mathrm{s}}(x)$ and $V_{\mathrm{a}}(x)$ are derived as

$$
V_{\mathrm{s}}(x) \equiv V_{\jmath \jmath}(x)=\frac{V(x)+V(-x)}{2}=\frac{x^{2}\left(x^{2}-2 a\right)}{4} ; V_{\mathrm{a}}(x) \equiv V_{\jmath \jmath^{\prime}}(x)=\frac{V(x)-V(-x)}{2}=b x^{3}, \jmath, \jmath^{\prime} \in\{0,1\}, \jmath \neq \jmath^{\prime}
$$

The matrix $W$ (cfr. (20) ) is calculated as $W_{y^{\prime}}=-V_{\mathrm{a}}\left(1-\delta_{\jmath, \jmath^{\prime}}\right)$ with the total rate represented by $W_{\jmath}=-V_{\mathrm{a}}$. The Euclidean-time Schrödinger equation [cfr. (19)] can thus be written

$$
\frac{\partial}{\partial \tau} K\left[x, \jmath, \tau ; x^{\prime}, \hat{\jmath}, 0\right]=\frac{1}{2} \frac{\partial^{2}}{\partial x^{2}} K\left[x, \jmath, \tau ; x^{\prime}, \hat{\jmath}, 0\right]-V_{\mathrm{s}}(x) K_{I}\left[x, \jmath, \tau ; x^{\prime}, \hat{\jmath}, 0\right]-\sum_{\jmath^{\prime}=0}^{1} V_{\mathrm{a}}(x) K\left[x, \jmath^{\prime}, \tau ; x^{\prime}, \hat{\jmath}, 0\right]\left(1-\delta_{\jmath, \jmath^{\prime}}\right) .
$$

Eq. (28) allows for a change of the boundary conditions of the propagator $K\left[x, \jmath, \tau ; x^{\prime}, \hat{\jmath}, 0\right]$ during its evolution in Euclidean time. Written as a Feynman-Kac functional, the propagator $K\left[x, \jmath, \tau ; x^{\prime}, \hat{\jmath}, 0\right]$ can be interpreted as a sum of two interdependent distinguishable-particle diffusion subprocesses. The numerical realization of the subprocesses $\jmath$ occurs by means of ensembles $\left(x_{i}^{\jmath}\right)_{i=1, n_{\jmath}}$ of $n_{\jmath}$ walkers. Each of the $n_{\jmath}$ walkers satisfies the boundary conditions for the subprocess $\jmath$ and during the evolution a walker may change its subprocess $\tilde{\jmath}$ to $\jmath$ according to the normalized transition amplitudes $p_{\jmath \rightarrow J^{\prime}}\left(\bar{r}_{f}\right)$. To formulate a probability-based evolution scheme it is therefore required that $p_{\jmath \rightarrow \jmath^{\prime}}\left(\bar{r}_{f}\right)$ remains positive. Since 


$$
\sum_{\bar{\jmath}=0}^{1} \exp \left(-\tau V_{J^{\prime} \bar{\jmath}}\left(\bar{r}_{f}\right)\right)=2 \exp \left(-\tau \frac{\left(x^{2}-a\right)^{2}-a^{2}}{4}\right)\left[\cosh \left(-\tau b x^{3}\right) \delta_{J^{\prime}, \jmath}+\sinh \left(-\tau b x^{3}\right)\left(1-\delta_{J^{\prime}, \jmath}\right)\right],
$$

the half-space $D^{-}$represents the appropriate state space for probabilistic sampling. Walker transitions to $x>0$ are prevented by the construction of a reflecting (for $\jmath=0$ ) or an absorbing (for $\jmath=1$ ) boundary. The major algorithmic steps for the numerical evolution procedure are enlisted as follows:

1. Generate two distinct walker sets $\left(x_{i}^{\jmath}\right)_{i=1, n_{\jmath}}, \jmath=0,1$. The initial non-zero walker populations $n_{\jmath}$ are chosen randomly.

2. Calculate for each walker the subprocess-transition probabilities $p_{\mathrm{a}}(x)=\left[1-\exp \left(-2 \Delta \tau b x^{3}\right)\right] / 2$ and apply them by comparison with uniform pseudo-random numbers $\eta \in[0,1]$ : if $\eta \leq p_{\mathrm{f}}(x)$ change the boundary condition associated to the walker, else leave the condition unchanged.

3. Make an evolution step, as indicated in Sec. A.

4. Return to step 1. until the density has equilibrated; after a certain amount of repetitions, calculate the potential average and the walker outflow to get an estimate for the ground-state energy.

5. To obtain an image of the sampled ground-state wave function, record the walker positions in small discrete bins.

The results obtained for the ground-state energy $E_{0}$ of the double-well system are shown in table 1 . To test the algorithm, the estimates $E_{0}^{\mathrm{MBDA}}$ have been compared with the numerical outcome $E_{0}^{\text {conf }}$ of a program with evolution on the full configuration space. For both the symmetric and the asymmetric potential, the results agree within the estimated standard deviation $\sigma$. It is illuminating to examine the population sizes of the two subprocesses $\jmath$. For the symmetric potential the probability $p_{\mathrm{a}}(x)$ is zero and independent subprocesses are simulated. Due to the higher eigenenergy related to the fermion subprocess $K\left[x, 0, \tau ; x^{\prime}, \hat{\jmath}, 0\right]$, the antisymmetric process rapidly fades out and the ground state wave function is completely described by the symmetric process. For the asymmetric potential in contrast, the antisymmetric subprocess is of crucial importance, since the asymmetric ground-state wave function cannot be simply generated by a symmetric process. The faster decay of the antisymmetric density is counteracted by a net walker transition from the symmetric to the antisymmetric population. In equilibrium, almost half of the population belongs to the antisymmetric density.

\begin{tabular}{l||c|c|c|c|c|c||} 
& $E_{0}^{\mathrm{conf}}$ & $\sigma^{\mathrm{conf}}$ & $E_{0}^{\mathrm{MBDA}}$ & $\sigma^{\mathrm{MBDA}}$ & $\sum \Psi_{\mathrm{s}} / \sum \Psi$ & $\sum \Psi_{\mathrm{a}} / \sum \Psi$ \\
\hline \hline$b=0$ & -0.2996 & 0.0006 & -0.2999 & 0.0006 & $100 \%$ & $0 \%$ \\
$b=0.25$ & -1.0251 & 0.0012 & -1.0251 & 0.0011 & $53.7 \%$ & $46.3 \%$ \\
\hline \hline
\end{tabular}

Table 1

This example for a particle in one dimension can be transparently generalized to arbitrary spatial dimensions. Consider, e.g., a particle in two dimensions exposed to the Mexican-Hat potential

$$
H=-\frac{1}{2} \frac{\partial^{2}}{\partial x^{2}}-\frac{1}{2} \frac{\partial^{2}}{\partial y^{2}}+V(x, y) \quad \text { with } \quad V(x, y)=\frac{\left(x^{2}+y^{2}-a\right)^{2}-a^{2}}{4}+b x^{3} .
$$

The two parameter sets $a=2, b=0$ and $a=2, b=0.25$ were chosen as in one dimension to represent a symmetric and an asymmetric potential, as depicted in fig. 2. Consider the two operators $\bar{P}_{x}: x \rightarrow-x$ and $\bar{P}_{y}: y \rightarrow-y$ and introduce the two boundaries $x=0$ and $y=0$. The construction of reflecting and absorbing boundary conditions at these two boundaries calls for the definition of 16 Euclidean-time propagators $K\left(x, \tau, \jmath ; x^{\prime}, \hat{\jmath}\right)$ and four elementary states $\Psi_{\mathrm{ss}}, \Psi_{\mathrm{sa}}, \Psi_{\mathrm{as}}$ and $\Psi_{\mathrm{aa}}$ with their indices characterizing their properties under transformation with $\bar{P}_{x}$ and $\bar{P}_{y}$, respectively. By imposing reflecting and absorbing boundary conditions the configuration space $\mathbb{R}^{2}$ is split into four subspaces with positive or negative $x$ - or $y$-coordinates. Although the system's evolution can be folded to any of these four subspaces, the analysis of the subprocess-transition amplitudes for the Mexican-Hat potential reveals that only two subspaces are associated with positive definite sampling. A sign analysis of the four subprocess-transition amplitudes $p_{\text {ss }}\left(\bar{r}_{f}\right), p_{\text {sa }}\left(\bar{r}_{f}\right), p_{\text {as }}\left(\bar{r}_{f}\right)$ and $p_{\text {aa }}\left(\bar{r}_{f}\right)$

$$
p_{\mathrm{as}}(x)=\frac{1-\exp \left(-2 \Delta \tau b x^{3}\right)}{2}, p_{\mathrm{ss}}(x)=\frac{1+\exp \left(-2 \Delta \tau b x^{3}\right)}{2}, p_{\mathrm{sa}}(x)=0, p_{\mathrm{aa}}(x)=0,
$$

indicates that they all may be interpreted as probabilities if the evolution is confined to either the subspace $D_{-}^{+} \equiv$ $\{x \leq 0, y \geq 0\}$ or $D_{-}^{-} \equiv\{x \leq 0, y \leq 0\}$. The fact that $p_{\mathrm{sa}}(x)=0$ and $p_{\mathrm{aa}}(x)=0$ induces two independent sets of 
subprocesses, namely $\left[K\left(x, \tau, \mathrm{ss} ; x^{\prime}, \hat{\jmath}\right), K\left(x, \tau\right.\right.$, as; $\left.\left.x^{\prime}, \hat{\jmath}\right)\right]$ and $\left[K\left(x, \tau, \mathrm{sa} ; x^{\prime}, \hat{\jmath}\right), K\left(x, \tau\right.\right.$,aa; $\left.\left.x^{\prime}, \hat{\jmath}\right)\right]$. Only those subprocess transitions are allowed which conserve the parity under $\bar{P}_{y}$. The formulation of the respective MBDA yields results for the ground-state energy which are in good agreement with the results achieved without the introduction of boundaries (see table 2). Since the characteristics of the model (29) allow for a distinguishable-particle treatment, the boundary-free algorithm reliably simulates the properties under investigation. The comparison of the estimated ground-state energies demonstrates the efficiency of the MBDA. Both energies $E_{0}^{\text {conf }}$ and $E_{0}^{\mathrm{MBDA}}$ have been obtained with comparable numerical effort and standard deviations. The last four columns of table 2 show the relevance of the four states $\Psi_{\mathrm{ss}}, \Psi_{\mathrm{sa}}, \Psi_{\mathrm{as}}$ and $\Psi_{\mathrm{aa}}$ with as measure of their relative population sizes. Since $p_{\mathrm{sa}}(x)=0$ and $p_{\mathrm{aa}}(x)=0$, the states $\Psi_{\mathrm{sa}}$ and $\Psi_{\mathrm{aa}}$ fade out. In addition, for the symmetric potential $p_{\mathrm{as}}(x)=0$. This means that also $\Psi_{\mathrm{as}}$ is irrelevant for the sampling of the ground-state wave function. The remaining state $\Psi_{\mathrm{ss}}$ is symmetric under both $\bar{P}_{x}$ and $\bar{P}_{y}$. Clearly, evolution in the presence of the asymmetric potential cannot be simulated with a symmetric state only; the algorithm ensures the occurrence of the $\Psi_{\text {as }}$ state by a steady transformation of walkers associated with the subprocess $K\left(x, \tau, \mathrm{ss} ; x^{\prime}, \hat{\jmath}\right)$ to walkers propagating according to $K\left(x, \tau\right.$, as; $\left.x^{\prime}, \hat{\jmath}\right)$.

\begin{tabular}{|c|c|c|c|c|c|c|c|c|}
\hline & $E_{0}^{\text {conf }}$ & $\sigma^{\text {conf }}$ & $\mathrm{C}_{0}^{\mathrm{MBDA}}$ & & $\Psi_{\mathrm{ss}} / \sum \Psi$ & $\Psi_{\mathrm{as}} / \sum \Psi$ & $\sum \Psi_{\mathrm{sa}} / \sum \Psi$ & \\
\hline & \begin{tabular}{|c|}
-0.19865 \\
\end{tabular} & 0.00042 & -0.19860 & 0.00037 & $\begin{array}{l}100 \% \\
\end{array}$ & $\begin{array}{c}0 \% \\
0 \%\end{array}$ & $0 \%$ & $\begin{array}{l}0 \% \\
0 \%\end{array}$ \\
\hline$b=0.25$ & -0.56129 & 0.00054 & -0.56115 & 0.00050 & $60.5 \%$ & $39.5 \%$ & $0 \%$ & $0 \%$ \\
\hline
\end{tabular}

Table 2

\section{Anisotropic Harmonic Fermions}

A system of two non-interacting anisotropic identical fermion oscillators in three dimensions serves as a transparent testing ground for indistinguishable particles. We considered a system with the Hamiltonian

$$
H=\frac{\bar{p}^{2}}{2}+\frac{\Omega_{x}^{2}}{2}\left(x_{1}^{2}+x_{2}^{2}\right)+\frac{\Omega_{y}^{2}}{2}\left(y_{1}^{2}+y_{2}^{2}\right)+\frac{\Omega_{z}^{2}}{2}\left(z_{1}^{2}+z_{2}^{2}\right),
$$

with ground-state energy

$$
E_{0}=\Omega_{x}+\Omega_{y}+\Omega_{z}+\operatorname{Min}\left[\Omega_{x}, \Omega_{y}, \Omega_{z}\right]
$$

We now consider a rotated reference frame as the result of from the rotation by the two Euler angles $\phi$ and $\theta[13]$

$$
\bar{R}=\left[\begin{array}{ccc}
\cos \phi & \sin \phi & 0 \\
-\sin \phi \cos \theta & \cos \phi \cos \theta & 0 \\
\sin \phi \sin \theta & -\cos \phi \sin \theta & \cos \theta
\end{array}\right] \bar{r} .
$$

The objectives are clear: the symmetry of the potential under the interchange of Cartesian particle coordinates is avoided without losing the advantage of comparison with a rigorous analytical solution. A proper implementation of the propagator with the MBDA requires positive definite subprocess transition amplitudes

$$
p_{\bar{l}}\left(\bar{r}_{f}\right)=\frac{\sum_{S_{x}} \sum_{S_{y}} \sum_{S_{z}} \xi_{l}^{S_{x}} \xi_{l}^{S_{y}} \xi_{l}^{S_{z}} \exp \left(-\tau V\left(S_{x} S_{y} S_{z} \bar{r}_{f}\right)\right) \xi_{\bar{l}}^{S_{x}} \xi_{\bar{l}}^{S_{y}} \xi_{\bar{l}}^{S_{z}}}{\sum_{\bar{l}} \sum_{S_{x}} \sum_{S_{y}} \sum_{S_{z}} \xi_{l}^{S_{x}} \xi_{l}^{S_{y}} \xi_{l}^{S_{z}} \exp \left(-\tau V\left(S_{x} S_{y} S_{z} \bar{r}_{f}\right)\right) \xi_{\bar{l}}^{S_{x}} \xi_{\bar{l}}^{S_{y}} \xi_{\bar{l}}^{S_{z}}},
$$

where we used the notation introduced in Sec. II. If the numerator of eq. (32) is positive definite, $p_{l \bar{l}}\left(\bar{r}_{f}\right)$ may be interpreted as the probability for a subprocess transition $\bar{l} \rightarrow l$. Since the sign of $p_{l \bar{l}}\left(\bar{r}_{f}\right)$ is determined by the parity of the propagator $K\left[\bar{r}_{f}, l, \Delta \tau ; \bar{r}_{i}, \hat{l}, 0\right]$ and the boundary condition $\bar{l}$ under Cartesian-coordinate permutation, four different subprocess-transition amplitudes occur. Their classification according to their symmetry properties suggests the following notation: $p_{\mathrm{bbb}}\left(\bar{r}_{f}\right), p_{\mathrm{ffb}}\left(\bar{r}_{f}\right), p_{\mathrm{fbf}}\left(\bar{r}_{f}\right)$ and $p_{\mathrm{bff}}\left(\bar{r}_{f}\right)$. The probability $p_{\mathrm{ffb}}\left(\bar{r}_{f}\right)$, e.g., stands for transitions between subprocesses with even parity in the $z$-coordinate and odd parity in the $x, y$-coordinates, for instance $K\left[\bar{r}_{f}, \mathrm{fbb}, \Delta \tau ; \bar{r}_{i}, \mathrm{bfb}, 0\right]$. While the amplitude $p_{\mathrm{bbb}}(\vec{r})$ is always positive, positivity of the three remaining amplitudes is imposed (in first order of the Euclidean time step $\Delta \tau$ ) by the following conditions on the domain and the parameters $\Omega_{x}, \Omega_{y}, \Omega_{z}, \phi$ and $\theta$ :

$$
\left\{\begin{array}{ll}
\sin \phi \cos \phi\left(\Omega_{x}^{2}-\Omega_{y}^{2} \cos ^{2} \theta+\Omega_{z}^{2}\left(\cos ^{2} \theta-1\right)\right)\left(x_{1}-x_{2}\right)\left(y_{1}-y_{2}\right) & \geq 0 \\
\cos \phi \sin \theta \cos \theta\left(\Omega_{y}^{2}-\Omega_{z}^{2}\right)\left(y_{1}-y_{2}\right)\left(z_{1}-z_{2}\right) & \geq 0 \\
-\sin \phi \sin \theta \cos \theta\left(\Omega_{y}^{2}-\Omega_{z}^{2}\right)\left(x_{1}-x_{2}\right)\left(z_{1}-z_{2}\right) & \geq 0
\end{array}\right\} .
$$


For the present illustration, the evolution has been restricted to the state space $\widehat{D}_{3}^{2}$ with a particular set of parameters:

$$
x_{1} \geq x_{2}, y_{1} \geq y_{2}, z_{1} \geq z_{2}, \Omega_{x}=4, \Omega_{y}=3, \Omega_{z}=2, \phi=130^{\circ}, \theta=50^{\circ} .
$$

Employing samples of approximately 20,000 walkers and Euclidean time steps of $0.001 \mathrm{H}^{-1}$, the MBDA leads to the numerical results visualized in fig. 5. The ground-state energy estimates (see left-hand side of fig. 5) oscillate in a small interval around their average of $10.9987 \pm 0.0056 \mathrm{H}$. They estimate the rigorous ground-state energy of 11.0 $\mathrm{H}$ with a statistical inaccuracy of about half a per mil. After numerical thermalization, the population of each of the four subprocesses $\{f, b, b\},\{b, f, b\},\{b, b, f\}$ and $\{f, f, f\}$ remains approximately constant (see right-hand side of fig. $5)$. In particular, contributions from the $\{f, f, f\}$-subprocess prove indispensable to correctly generate the propagator. Our numerical investigations manifested the occurrence of a net walker flow from the subdensities $\Psi_{\mathrm{fbb}}, \Psi_{\mathrm{bfb}}, \Psi_{\mathrm{bbf}}$ to $\Psi_{\text {fff. }}$. Without the inclusion of subprocess interdependencies, the subprocess $K\left[\bar{r}_{f}\right.$, fff, $\left.\Delta \tau ; \bar{r}_{i}, \hat{l}, 0\right]$ would fade out exponentially in evolution time.

\section{Application to Ortho Helium}

To illustrate the feasibility of the reduced state space concept, we also used the MBDA to calculate the groundstate energy of ortho-helium. In the triplet states of ortho-helium, the two electrons carry equal spin. The occurrence of additional repulsive forces due to exchange distinctly influences the energy spectrum, so that the simulation of the lowest ortho-helium state requires the accurate inclusion of Fermi statistics. In that context, the challenge of numerical implementation shows twofold. First, the lowest energy level should be reliably predicted and second, the converged energy estimates should be stable in time. In particular the second aspect concerns the fermion sign problem: the MBDA is demonstrated to exactly filter out possible interfering symmetric contributions. The general evolution scheme on the reduced state space $\widehat{D}_{2}^{3}$ relies on the construction of $32^{2}$ interdependent subprocesses $K\left[\bar{r}_{f}, l, l^{\prime}, \Delta \tau ; \bar{r}_{i}, \hat{l}, \hat{l}^{\prime}, 0\right]$. Here, each of the $l, \hat{l}$ may be one of the four boundary conditions fff,fbb,bfb or bbf, whereas $l^{\prime}, \hat{l}^{\prime}$ run over the eight conditions bbb,fbb,..,fff. Symmetry arguments allow to simplify the computational procedure by the prior rejection of certain classes of subprocesses. As the permutation operator $P$ has the same effect on the ortho-helium Hamiltonian as the permutation operator $\widehat{P}$, the ground-state wave function consists of substates with equal parity under $P$ and $\widehat{P}$. Consequently, the simulation of the ortho-helium ground-state wave function on the reduced state space $\widehat{D}_{2}^{3}$ is accomplished by the construction of only 16 subprocesses with $l=\hat{l}, l^{\prime}=\hat{l}^{\prime}$ specified as $\mathrm{fff}, \mathrm{fbb}, \mathrm{bfb}$ or bbf. Although both - the general and the simplified - procedure in principle lead to the exact densities, the restriction to four subprocesses considerably improves the convergence of the algorithm. The next step in the formulation of an evolution scheme for the ortho-helium ground state is the consideration of the subprocess-transition amplitudes

$$
p_{l \bar{l}}^{l^{\prime} \bar{l}^{\prime}}\left(\bar{r}_{f}\right)=\frac{\sum_{\widehat{S}_{x}, \widehat{S}_{y}, \widehat{S}_{z}, \widehat{S}_{x}, \widehat{S}_{y}, \widehat{S}_{z}} \xi_{l}^{\widehat{S}_{x}} \xi_{l}^{\widehat{S}_{y}} \xi_{l}^{\widehat{S}_{z}} \xi_{l^{\prime}}^{S_{x}} \xi_{l^{\prime}}^{S_{y}} \xi_{l^{\prime}}^{S_{z}} e^{-\tau V\left(\widehat{S}_{x} \widehat{S}_{y} \widehat{S}_{z} S_{x} S_{y} S_{z} \bar{r}_{f}\right)} \xi_{\bar{l}}^{S_{x}} \xi_{\bar{l}}^{S_{y}} \xi_{\bar{l}}^{S_{z}} \xi_{\bar{l}^{\prime}} \widehat{S}_{x} \widehat{S}_{\bar{l}^{\prime}} \widehat{S}_{\bar{l}^{\prime}} \widehat{S}_{z}}{\sum_{\bar{l}, \bar{l}^{\prime}, \widehat{S}_{x}, \widehat{S}_{y}, \widehat{S}_{z}, \widehat{S}_{x}, \widehat{S}_{y}, \widehat{S}_{z}} \xi_{l}^{\widehat{S}_{x}} \xi_{l}^{\widehat{S}_{y}} \xi_{l}^{\widehat{S}_{z}} \xi_{l^{\prime}}^{S_{x}} \xi_{l^{\prime}}^{S_{y}} \xi_{l^{\prime}}^{S_{z}} e^{-\tau V\left(\widehat{S}_{x} \widehat{S}_{y} \widehat{S}_{z} S_{x} S_{y} S_{z} \bar{r}_{f}\right)} \xi_{\bar{l}}^{S_{x}} \xi_{\bar{l}}^{S_{y}} \xi_{\bar{l}}^{S_{z}} \xi_{\bar{l}^{\prime}} \widehat{S}_{x} \xi_{\bar{l}^{\prime}}^{\widehat{S}_{y} \widehat{S}_{\bar{l}_{z}}} \xi_{\bar{l}^{\prime}}}
$$

controlling the interplay between the four single subprocesses. In order to derive the subspaces associated with positive sums of exponentials $e^{-\tau V\left(\widehat{S}_{x} \widehat{S}_{y} \widehat{S}_{z} S_{x} S_{y} S_{z} \bar{r}_{f}\right)}$, we focus on the short-time limits $\left(1-\tau V\left(\widehat{S}_{x} \widehat{S}_{y} \widehat{S}_{z} S_{x} S_{y} S_{z} \bar{r}_{f}\right)\right)$ of the exponentials, which contain all the essential symmetry characteristics necessary for the sign analysis of the amplitudes (35). Furthermore, it proves useful to separate the potential $V(\bar{r})$ in two parts $V_{1}(\bar{r})$ and $V_{2}(\bar{r})$ with

$$
V_{1}(\bar{r})=\frac{-2}{\left|\vec{r}_{1}\right|}+\frac{-2}{\left|\vec{r}_{2}\right|} \quad, \quad V_{2}(\bar{r})=\frac{1}{\left|\vec{r}_{1}-\vec{r}_{2}\right|} \quad, \quad V(\bar{r})=V_{1}(\bar{r})+V_{2}(\bar{r}) .
$$

To illustrate the need of a reduced state space, consider the corresponding many-body diffusion process on the state space $D_{2}^{3}$. The amplitudes $p_{\mathrm{bbb}}(\bar{r}), p_{\mathrm{ffb}}(\bar{r}), p_{\mathrm{fbf}}(\bar{r})$ and $p_{\mathrm{bff}}(\bar{r})$ control the transitions between the 16 fermion subprocesses $K\left[\bar{r}_{f}, l, \Delta \tau ; \bar{r}_{i}, \hat{l}, 0\right]$. A detailed investigation of the amplitudes, however, discloses that some of them may become negative on the state space $D_{2}^{3}$. For instance, considering $p_{\mathrm{ffb}}(\bar{r})$, one derives

$$
\begin{aligned}
& p_{\mathrm{ffb}}(\bar{r}): \stackrel{\tau \rightarrow 0}{\longrightarrow} e^{\tau V(\bar{r})} \sum_{P_{x}, P_{y}, P_{z}}(-)^{P_{x}}(-)^{P_{y}}\left(1-V_{1}\left(P_{x} P_{y} P_{z} \bar{r}\right)\right) \\
\propto & \frac{4 \tau}{\sqrt{\pi}} \int_{-\infty}^{\infty} d t\left(e^{-x_{1}^{2} t^{2}}-e^{-x_{2}^{2} t^{2}}\right)\left(e^{-y_{1}^{2} t^{2}}-e^{-y_{2}^{2} t^{2}}\right)\left(e^{-z_{1}^{2} t^{2}}+e^{-z_{2}^{2} t^{2}}\right)
\end{aligned}
$$


which is positive definite on a subdomain of the state space, namely for $\left|x_{1}\right| \geq\left|x_{2}\right|$ and $\left|y_{1}\right| \geq\left|y_{2}\right|$. Similar restrictions follow from $p_{\mathrm{fbf}}(\bar{r})$ and $p_{\mathrm{bff}}(\bar{r})$, whereas $p_{\mathrm{bbb}}(\bar{r})$ is a sum of only positive elements. Strictly speaking, utilizing the state space as the domain for evolution, the MBDA would incorrectly simulate the ortho-helium propagator. It is at that point that the reduced state space $\widehat{D}_{2}^{3}$ as defined by (24) comes into play. By appropriate restriction of the evolution to the reduced state space, the subprocess-transition amplitudes all remain positive and their probabilistic implementation is justified. In what follows, we take over the results from Sec. III and adapt the general scheme to the ortho-helium system.

Continuing with the investigation of the amplitudes $p(\bar{r})$, let us first focus on the contributions of $V_{1}(\bar{r})$. In $V_{1}(\bar{r})$, the coordinates $x_{1}, \ldots, z_{2}$ are entangled such that the transformations $P$ and $\hat{P}$ have the same effects; for our purpose, no distinction has to be made between $P$ and $\hat{P}$.

With regard to amplitudes with identical sub- and superindices per coordinate, one obtains

$$
\begin{aligned}
\left.p_{\mathrm{ffb}}^{\mathrm{ffb}}(\bar{r})\right|_{V_{1}} & =e^{\tau V(\bar{r})} \sum_{P_{x}, P_{y}, P_{z}, \widehat{P}_{x} \widehat{P}_{y}, \widehat{P}_{z}}\left(-P^{P_{x}}(-)^{P_{y}}(-)^{\widehat{P}_{x}}(-)^{\widehat{P}_{y}} \exp \left(P_{x} P_{y} \widehat{P}_{x} \widehat{P}_{y} \bar{r}\right)\right. \\
& : \stackrel{\tau \rightarrow 0}{\longrightarrow} \frac{\tau}{2 \sqrt{\pi}} e^{\tau V_{1}(\bar{r})} \int_{-\infty}^{\infty} d t\left(e^{-x_{1}^{2} t^{2}}-e^{-x_{2}^{2} t^{2}}\right)\left(e^{-y_{1}^{2} t^{2}}-e^{-y_{2}^{2} t^{2}}\right)\left(e^{-z_{1}^{2} t^{2}}+e^{-z_{2}^{2} t^{2}}\right) .
\end{aligned}
$$

The remaining 28 amplitudes have in common that for at least one coordinate the permutations $P$ and $\widehat{P}$ induce different parity. Each of these subprocess-transition amplitudes involves a sum composed of two parts of equal absolute value but opposite sign. Consequently, they are irrelevant for the evolution procedure.

The potential $V_{2}(\bar{r})$ proves invariant under interchange of two coordinates. Its symmetry properties entail that only those amplitudes $p(\bar{r})$ contribute which in each direction involve equal positive (bosonlike) parity under $P$ and $\widehat{P}$, i.e.

$$
\left.p_{\mathrm{bbb}}^{\mathrm{bbb}}(\bar{r})\right|_{V_{2}} \propto \sum_{P_{x}, P_{y}, P_{z}, \widehat{P}_{x} \widehat{P}_{y}, \widehat{P}_{z}} \exp \left(-\tau P_{x} P_{y} P_{z} \widehat{P}_{x} \widehat{P}_{y} \widehat{P}_{z} \bar{r}\right): \stackrel{\tau \ll 1}{\longrightarrow} \exp \left(-\tau V_{2}(\bar{r})\right)
$$

Obviously, $p_{\mathrm{bbb}}^{\mathrm{bbb}}(\bar{r})$ is positive on the reduced state space $\widehat{D}_{2}^{3}$. By recombination of both potentials $V_{1}(\bar{r})$ and $V_{2}(\bar{r})$, the total evolution scheme is established. Its proper implementation guarantees accurate probabilistic sampling of the ortho-helium propagator on the reduced state space $\widehat{D}_{2}^{3}$. The sampled density consists of 4 subdensities $\Psi_{\mathrm{fbb}}^{\mathrm{fbb}}(\bar{r}, \tau), \Psi_{\mathrm{bfb}}^{\mathrm{bfb}}(\bar{r}, \tau), \Psi_{\mathrm{bbf}}^{\mathrm{bbf}}(\bar{r}, \tau)$ and $\Psi_{\mathrm{fff}}^{\mathrm{fff}}(\bar{r}, \tau)$. Due to the symmetry properties of the ortho-helium potential $V(\bar{r})=$ $V_{1}(\bar{r})+V_{2}(\bar{r})$, certain classes of subprocess transitions are forbidden; each of the subprocesses undergoes one out of four possible transitions ruled by the probabilities $p_{\mathrm{ffb}}^{\mathrm{fb}}(\bar{r}), p_{\mathrm{fbf}}^{\mathrm{fbf}}(\bar{r}), p_{\mathrm{bff}}^{\mathrm{bff}}(\bar{r})$ and $p_{\mathrm{bbb}}^{\mathrm{bbb}}(\bar{r})$. During a time step $\Delta \tau$, the subdensity $\Psi_{\mathrm{fbb}}^{\mathrm{fbb}}(\bar{r}, \tau)$, for instance, branches into the subdensities $\Psi_{\mathrm{bfb}}^{\mathrm{bbb}}(\bar{r}, \tau+\Delta \tau), \Psi_{\mathrm{bbf}}^{\mathrm{bbf}}(\bar{r}, \tau+\Delta \tau), \Psi_{\mathrm{fff}}^{\mathrm{fff}}(\bar{r}, \tau+\Delta \tau)$ and $\Psi_{\mathrm{fbb}}^{\mathrm{fbb}}(\bar{r}, \tau+\Delta \tau)$ according to the probabilities $p_{\mathrm{fbb}}^{\mathrm{fbb}}(\bar{r}), p_{\mathrm{fbf}}^{\mathrm{fbf}}(\bar{r}), p_{\mathrm{bff}}^{\mathrm{bff}}(\bar{r})$ and $p_{\mathrm{bbb}}^{\mathrm{bbb}}(\bar{r})$. Notice that the analysis of the subprocess transition amplitudes is consistent with the restriction to four subdensities in the sense that no transitions occur to subdensities different from the four specified ones. Fig. 6 illustrates the energy estimates for the lowest orthohelium state achieved with a pre-thermalized sample. The numerical average $-2.1744 \pm 0.0012 \mathrm{H}$ is in good agreement with the numerical energy estimate of $-2.175229 \mathrm{H}$ found in [14]. Systematic errors due to time discretization and imperfect sampling could be diminished by the use of total sample sizes of 100,000 walkers and time steps of 0.001 $\mathrm{H}^{-1}$.

\section{DISCUSSION AND CONCLUSIONS}

It should be mentioned that the relation between fermi statistics and the elimination of paths at permutation hyperplanes has been put forward by Korzeniowski et al. [15]. This approach was subsequently criticized [16], because it left open a few fundamental questions. Studying the underlying mathematical Ansatz has lead us to an extension of the recently reported many-body diffusion approach [2 5] in which it was the basic idea to rigorously separate the multi-dimensional free diffusion process of indistinguishable particles into one-dimensional free diffusion processes. The novelty of the outlined symmetry analysis for the simulation of indistinguishable particles is the construction of numerically implementable diffusion process with a switching of boundary conditions driven by a compound Markov chain . The continuous-time Markov chain, not considered before in that framework, has been set up to enable the realization of interdependent Brownian motions. The inclusion of transitions between a set of simultaneously evolving diffusion processes has been shown necessary to approach identical particles in potentials which are not invariant under permutations of Cartesian coordinates. To transfer the recently reported MBDA sampling technique [1] to those systems, a symmetry analysis of the potential is necessary. In applications to different model systems, the consequences 
for the total diffusion process have been demonstrated. The numerical findings confirmed the feasibility of the utilized symmetry concept. The model of one particle exposed to a double-well or a Mexican-Hat potential transparently illustrates the essence of our approach. Artificially creating a sign problem by the decomposition of the propagator in two resp. four subpropagators, some of which are not positive definite on the configuration space, the efficiency of the algorithm has been found comparable to the corresponding boundary-free formulation. This application points out the generality of the symmetry analysis on which the algorithm is founded; its use is not restricted to the description of identical particles but may also be of relevance for the implementation of boundary conditions on distinguishable particle systems. In the MBDA, the boundaries guarantee the generation of symmetric and antisymmetric Brownian motions. This is fundamentally different from nodal approaches; obviously, the ground-state wave function of the particle does not vanish at the boundaries (cfr. figs. 2 and 4)! The flexibility of the many-body diffusion Ansatz has been indicated by treating two identical fermion oscillators in three dimensions with an algorithm based on the same principles but different subprocess-transition probabilities. Evidence has been given that the MBDA avoids the fermion sign problem in applications to the ortho-helium model. Necessary ingredients for this study have been the derivation of the appropriate reduced state space $\widehat{D}_{2}^{3}$ with the properly adapted stochastic processes. The comparison of the numerical ground-state estimates indicated that the MBDA reliably simulates the exchange contributions. Over the considered evolution intervals no significant decay in the signal-to-noise ratio has been observed. The reduced state space boundary $\partial \widehat{D}_{2}^{3}$ does definitely not represent a nodal surface. Also in numerical practice, the characteristic fermion state is completely determined by the Euclidean-time Schrödinger equation supplied with the symmetry properties of the free-diffusion process and the potential under consideration. From that point of view, the MBDA represents a potential candidate for ab-initio fermion Monte Carlo simulations. In comparison with literature the MBDA - in its present form - is restricted by its versatility. The MBDA tackles fundamental methodological aspects to avoid the sign problem for fermions. To transparently demonstrate that the supply of nodal surfaces is unnecessary for the current approach, the present formulation dispenses with such analytical assumptions. We are well aware that importance sampling may drastically enhance computational efficiency, but the objective has been to keep the algorithm as transparent as possible. The nodal structure of the ortho-helium ground state, for instance, can be derived by symmetry arguments [17. Implementing an importance function with this nodal structure enables one to circumvent the sign problem. The MBDA algorithm, however, does not require to build in the nodal structure. We stress that the MBDA is fundamentally different from approaches based on interacting or correlated walker ensembles 18 22]. Dealing with many-fermion diffusion on the appropriate state space $D$, the ground-state wave function is simulated by a sum of antisymmetric elementary densities. This is in contrast to the common definition of positive and negative walker ensembles, the densities of which behave naturally symmetric. The MBDA involves only positive walkers. Antisymmetry is realized by establishing the correct free diffusion process without requiring projection by an antisymmetric trial function. The use of reflection and absorption restricts the evolution to the state space; this procedure proves useful as long as the supplementary effort for reflection and absorption is balanced by enhanced accuracy due to restriction to the state space. The present investigations mainly attempted to solve and clarify the fundamental difficulties related to the sign problem for fermions. The underlying symmetry concepts were analyzed and used to guarantee the stable simulation of fermion systems. Some transparent examples were chosen mainly for illustrative purpose and no inference has been made to provide ultimate accuracy.

\section{ACKNOWLEDGMENTS}

Work supported by the "PHANTOMS onderzoeksnetwerk" and Interuniversitaire Attractiepolen - Belgische Staat, Diensten van de Eerste Minister - Wetenschappelijke, technische en culturele aangelegenheden. We acknowledge support by the BOF NOI 1997 projects of the Universiteit Antwerpen, the FWO projects G.0071.98 and WO.073.94N (Wetenschappelijke Onderzoeksgemeenschap, Scientific Research Community of the FWO on "Low-Dimensional Systems"). Part of this work has been performed in the framework of the projects G.0287.95, 1.5.545.98 and the supercomputer project 1.5.729.94N of the FWO. F.L. is very grateful to B. Gerlach for partial financial support during the development of this work.

[1] F. Luczak, F. Brosens, J.T. Devreese, and L.F. Lemmens, Many-body diffusion algorithm: Harmonic fermions, accepted for publication in Phys. Rev. E.

[2] L.F. Lemmens, F. Brosens, and J.T. Devreese, Phys. Lett. A, 189, 473 (1994). 
[3] F. Brosens, J.T. Devreese, and L.F. Lemmens, Sol. State. Comm. 96, 137 (1995).

[4] L.F. Lemmens, F. Brosens, and J.T. Devreese, Phys. Rev. E 53, 4467 (1996).

[5] L.F. Lemmens, F. Brosens, and J.T. Devreese, Phys. Rev. E 55, 7813 (1997).

[6] M. Suzuki, Quantum Monte Carlo Methods, Solid State Sciences 74, ed. M. Suzuki, Springer, Berlin 1986; H. De Raedt and D. De Raedt, Phys. Rev. A 28, 3575 (1983); H. De Raedt, Comp. Phys. Rep. 7,1 (1987).

[7] D.M. Ceperley, Rev. Mod. Phys. 67, 279 (1995).

[8] J.M. Harrison, Brownian Motion and Stochastic Flow Systems, J. Wiley \& Sons, New York, 1985.

[9] N. Makri and W.H. Miller, Chem. Phys. Lett. 151, 1 (1988).

[10] D. Williams, and L. C. Rogers, Markov processes and martingales Vol II, J. Wiley \& Sons, New York, 1987.

[11] K.L. Chung and Zhongxin Zhao, From Brownian motion to Schrödinger equation, Springer, Berlin 1995;

[12] F. Cooper, A. Khare and U. Sukhatne, Phys. Rep. 251, 267 (1995)

[13] H. Goldstein, Classical Mechanics, Reading, Mass.

[14] C.J. Umrigar, K.E. Wilson, and J.W. Wilkins, in Computer Simulation Studies in Condensed Matter Physics: Recent Developments, ed. D.P. Landau, K.K. Mon and H.B. Schuttler, Springer, Berlin, 1988.

[15] A. Korzeniowski, J.L. Fry, D.B. Orr and N.G. Fazleev, Phys. Rev. Lett. 69, 893 (1992); ibid. 71, 2160 (1993).

[16] W.M.C. Foulkes, Phys. Rev. Lett. 71, 2158 (1993); M. Caffarel, D.M. Ceperley and M.H. Kalos, Phys. Rev. Lett. 71, 2159 (1993).

[17] D.J. Klein and H.M. Picket, J. Chem. Phys. 64, 4811 (1976).

[18] D.M. Arnow, J.H. Kalos, M.A. Lee, and K.E. Schmidt, J. Chem. Phys. 77, 5562 (1982).

[19] J.B. Anderson, C.A. Traynor, and B.M. Boghosian, J. Chem. Phys. 95, 7418 (1991).

[20] S. Zhang and M.H. Kalos, Phys. Rev. Lett. 67, 3074 (1991).

[21] Z. Liu, S. Zhang, and M.H. Kalos, Phys. Rev. E 50, 3220 (1994).

[22] M.H. Kalos, Phys. Rev. E 53, 5420 (1996).

\section{Figure CAPtions}

Fig. 1: The rigorous (right hand side) and numerically sampled (left hand side) Green function of two free identical fermions in one dimension restricted to the reduced state space $x_{1} \geq\left|x_{2}\right|$ after an evolution of three atomic time units. The initial condition is denoted by the crosses, the state-space boundaries are indicated by the solid lines.

Fig. 2: Plot of the symmetric and asymmetric double well potential $V_{s}(x)$ and $V_{a}(x)$ (27) with $a=2$. Solid line: $b=0$, dashed line: $b=0.25$. The long-dashed resp. dot-dashed curve depicts the corresponding simulated ground-state wave functions $\Psi_{s}(x)$ and $\Psi_{a}(x)$.

Fig. 3: Plot of the Mexican Hat potential (29) with $a=2$ and $b=0$ (l.h.s.) and with $a=2$ and $b=0.25$ (r.h.s.).

Fig. 4: Comparison of the two numerically predicted ground-state wavefunctions of the asymmetric Mexican-Hat model (29), with $a=2$ and $b=0.25$ sampled with the MBDA (l.h.s) and the boundary-free algorithm.

Fig. 5: The ground-state energy estimates (left hand side) and the relative walker population of the subdensities (right hand side) as a function of Euclidean evolution time for the three-dimensional anisotropic system of two identical fermion oscillators.

Fig. 6: Comparison of the numerical energy estimates (solid curve) for the lowest ortho-helium state with the numerical estimate $-2.175229 \mathrm{H}$ found in 14] (dashed curve). The dot-dashed lines denote a deviation of one percent from the dashed curve.

Tab. 1: Estimated ground-state energies $E_{0}$ with standard deviations $\sigma$ and relative subprocess population for the double-well potential potential (27).

Tab. 2: Same as table 1 but for the Mexican-Hat potential (29). 

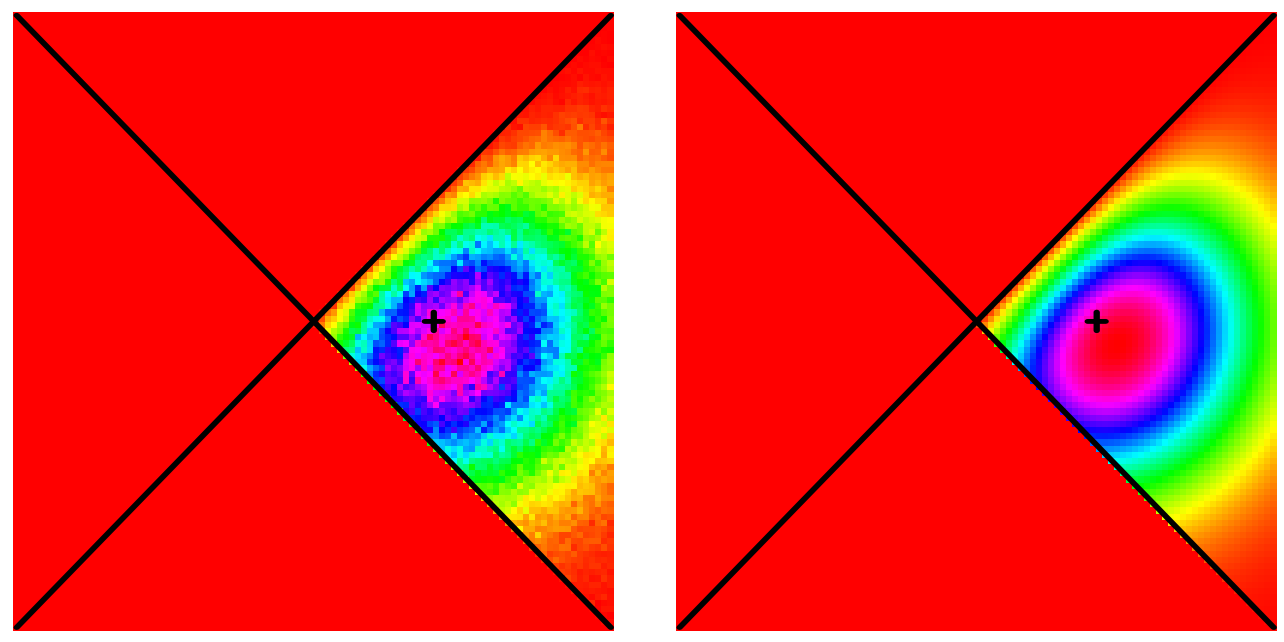

Fig.1 


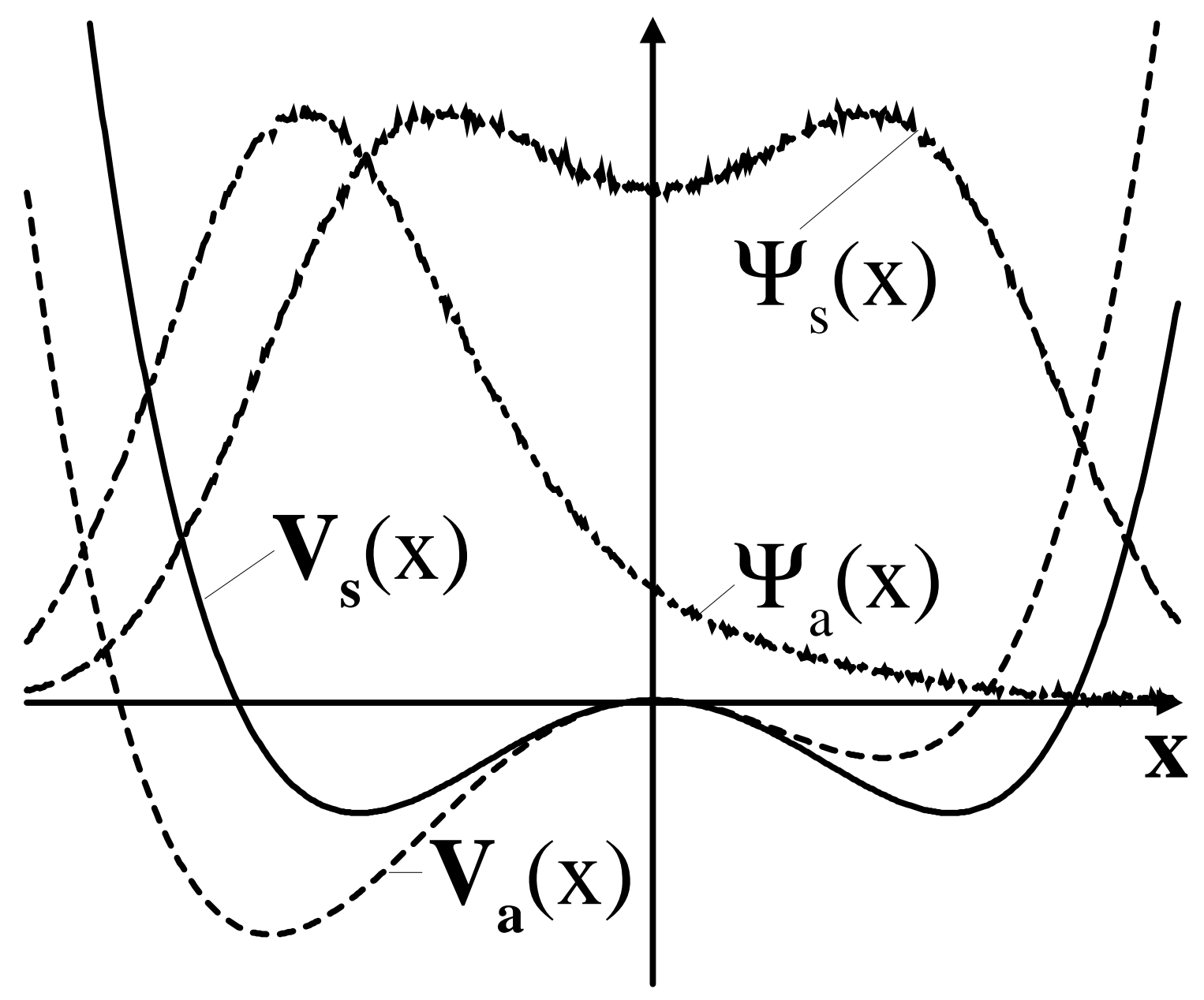



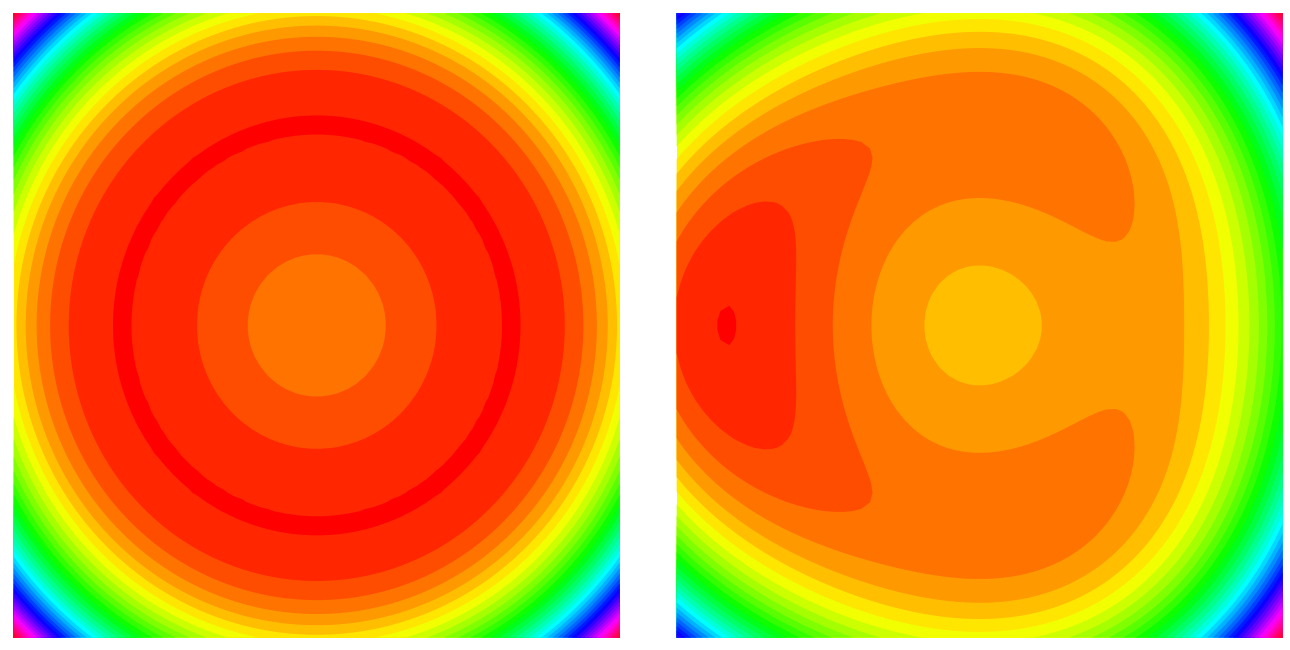

Fig.3 

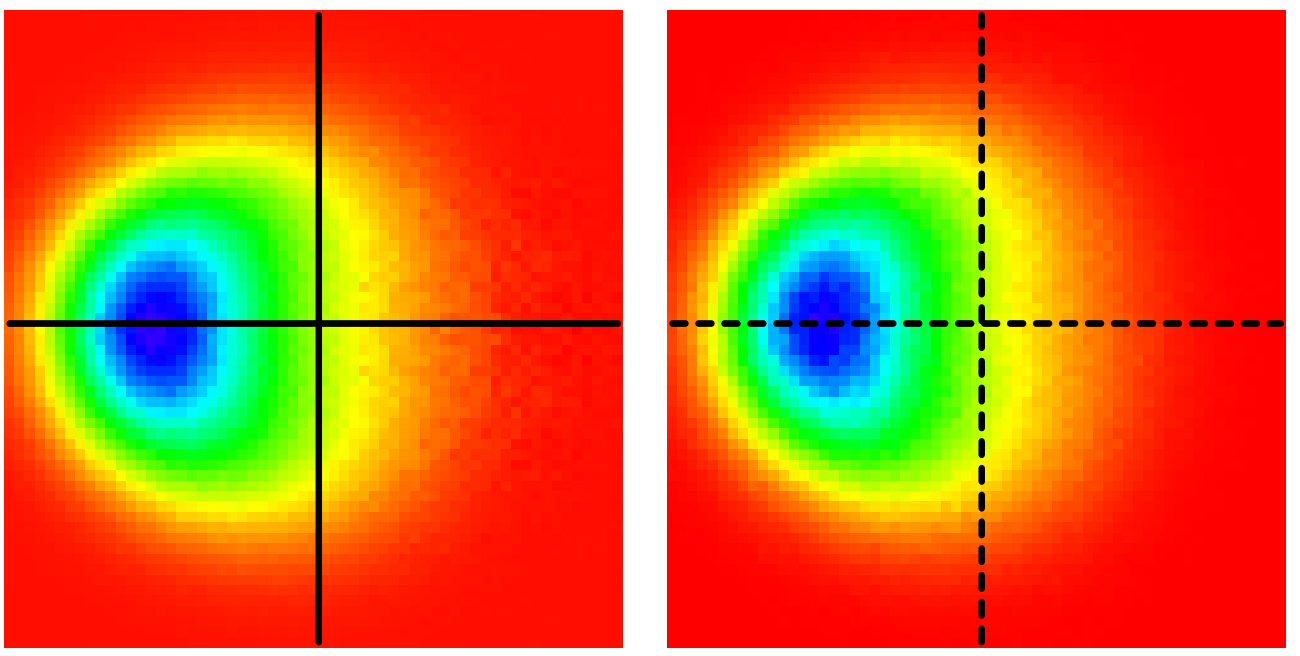

Fig.4 

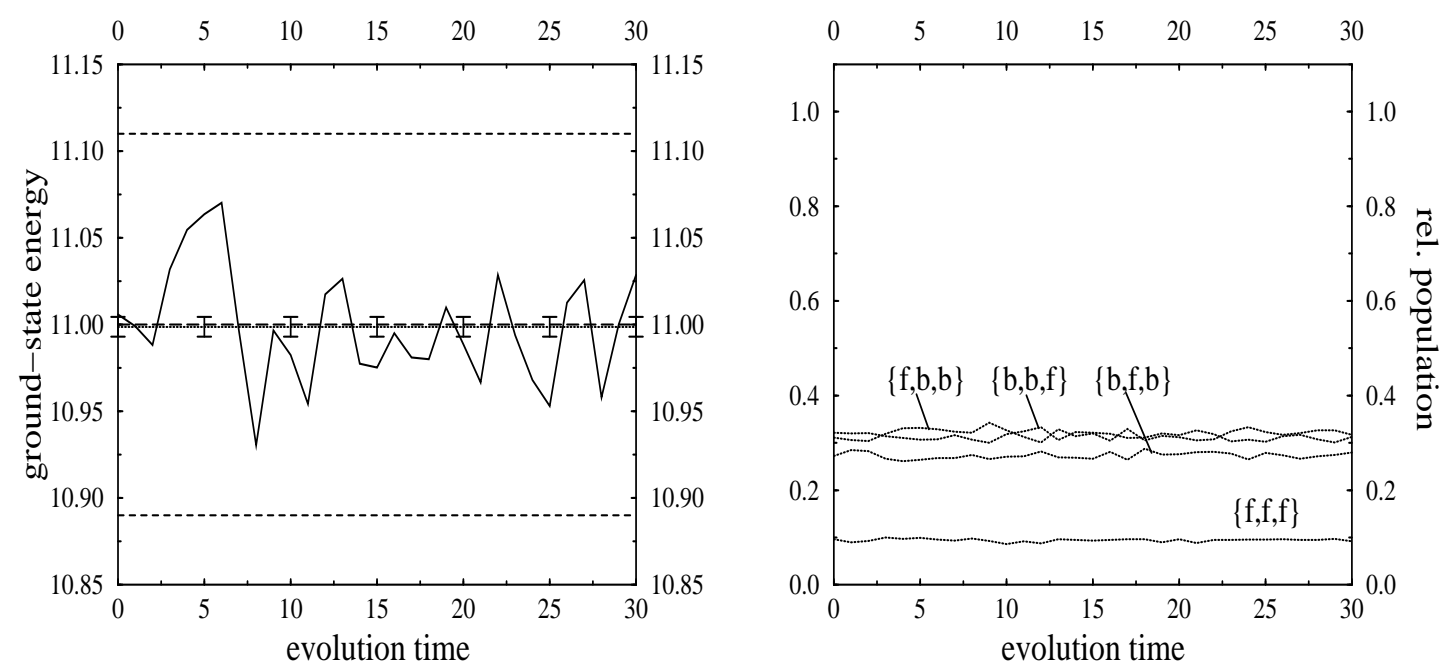

Fig.5 


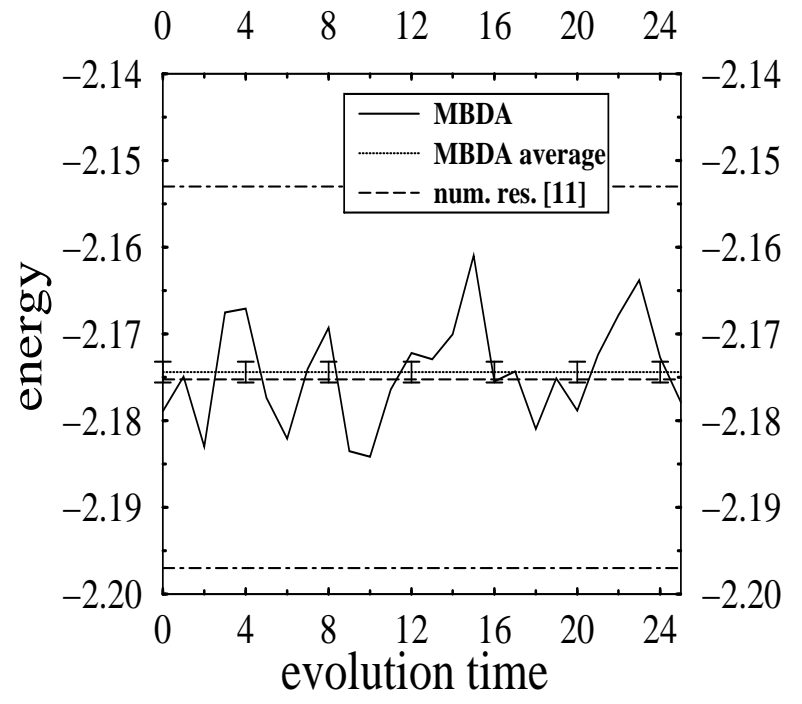

Fig.6 